\title{
BIFURCATION DIAGRAMS AND GLOBAL PHASE PORTRAITS FOR SOME HAMILTONIAN SYSTEMS WITH RATIONAL POTENTIALS
}

\author{
TING CHEN ${ }^{1,2}$ AND JAUME LLIBRE ${ }^{2, *}$
}

\begin{abstract}
In this paper we study the global dynamical behavior of the Hamiltonian system $\dot{x}=H_{y}(x, y), \dot{y}=-H_{x}(x, y)$ with the rational potential Hamiltonian $H(x, y)=y^{2} / 2+P(x) / Q(y)$, where $P(x)$ and $Q(y)$ are polynomials of degree 1 or 2 . First we get the normal forms for these rational Hamiltonian systems by some linear change of variables. Then we classify all the global phase portraits of these systems in the Poincaré disk and provide their bifurcation diagrams.
\end{abstract}

\section{IntRoduction AND STATEMENT OF THE MAIN RESUlts}

A great deal of work has been done for studying the global dynamics of the planar polynomial differential systems, for example see $[3,4,6,8,10,15]$. Vulpe [16] studied the global phase portraits of the quadratic polynomial systems having a center. In [14] Schlomiuk gave the bifurcation diagrams for the global phase portraits of these quadratic systems. Artés and Llibre [2] provided the global phase portraits of all quadratic Hamiltonian systems. The authors of [12] presented the phase portraits of the quadratic polynomial vector fields having a rational first integral of degree 3. Guillamon et al. [11] gave an algorithm to obtain the phase portraits of the separable Hamiltonian system with the Hamiltonian function $H(x, y)=F(x)+G(y)$. Colak et al. [5, 7] presented the global phase portraits in the Poincaré disk of all Hamiltonian linear type centers of polynomial systems having linear plus cubic homogeneous terms, and gave their bifurcation diagrams.

In this paper we consider the Hamiltonian system

$$
\dot{x}=H_{y}(x, y), \quad \dot{y}=H_{x}(x, y)
$$

with a rational Hamiltonian function

$$
H=H(x, y)=\frac{y^{2}}{2}+V(x, y)=\frac{y^{2}}{2}+\frac{P(x)}{Q(y)},
$$

where $P(x)$ and $Q(y)$ are real polynomials of degree at most 2 . We denote by the set $L=\{(x, y) \mid Q(y)=0\}$ the points where the Hamiltonian vector field are not defined. The system associated to the Hamiltonian function (2) has the form

$$
\dot{x}=y-\frac{P(x) Q^{\prime}(y)}{Q^{2}(y)}, \quad \dot{y}=-\frac{P^{\prime}(x)}{Q(y)},
$$

2010 Mathematics Subject Classification. Primary: 34C07, 34C08.

Key words and phrases. Rational Hamiltonian system, singularities, infinity, phase portrait, bifurcation diagram. 
where $P^{\prime}(x)$ and $Q^{\prime}(y)$ indicate the derivatives of the polynomials $P$ and $Q$ with respect to $x$ and $y$ respectively, and the dot denotes derivative with respect to the time $t$. Under the time rescaling

$$
\frac{d t}{d \tau}=Q^{2}(y)
$$

the rational Hamiltonian system (3) becomes the polynomial system

$$
x^{\prime}=y Q^{2}(y)-P(x) Q^{\prime}(y), \quad y^{\prime}=-P^{\prime}(x) Q(y),
$$

where $x^{\prime}$ and $y^{\prime}$ denote derivatives of $x$ and $y$ with respect to $\tau$ respectively. But the new system (5) is not Hamiltonian in general, we call it an integrable nonHamiltonian system. Thus we can study the phase portraits of the Hamiltonian (3) analyzing the associated polynomial differential system (5). Martínez and Vidal [13] classified the phase portraits of the Hamiltonian system (1) with the potential $V(x, y)=P(x) / Q(x)$. Our main result is the following one.

Theorem 1.1. Given a Hamiltonian (2) with the rational potential $V(x, y)=\frac{P(x)}{Q(y)}$ where $P(x)$ and $Q(y)$ are polynomials of degree at most 2, after a linear change of variables and a rescaling of its independent variables, it can be written as one of the following classes:

(I) $H_{11}^{ \pm}=\frac{y^{2}}{2} \pm \frac{x+\widetilde{b}}{y+B}$, with $\widetilde{b}=b /(a \sqrt{|a|})$ and $\widetilde{B}=B / \sqrt{|a|}$, if $P(x)=a x+b$ and $Q(y)=A y+B$ with $a A \neq 0$;

(II) $H_{21}^{ \pm}=\frac{y^{2}}{2} \pm \frac{x^{2} \pm \Delta}{y+B}$, with $\Delta=c-b^{2} /(4 a)$, if $P(x)=a x^{2}+b x+c$ and $Q(y)=A y+B$ with $a A \neq 0$;

(III) $H_{12}=\frac{y^{2}}{2}+\frac{x+\widetilde{b}}{y^{2}+\widetilde{B} y+\widetilde{C}}$, with $\widetilde{b}=b /(a \sqrt[3]{a}), \widetilde{B}=B / \sqrt[3]{a}$ and $\widetilde{C}=C / \sqrt[3]{a^{2}}$, if $P(x)=a x+b$ and $Q(y)=A y^{2}+B y+C$ with $a A \neq 0$;

(IV) $H_{22}^{ \pm}=\frac{y^{2}}{2} \pm \frac{x^{2}+\Delta}{y^{2}+\widetilde{B} y+\widetilde{C}}$, with $\Delta=\left(c / a-b^{2} /\left(4 a^{2}\right)\right) /|a|, \widetilde{B}=B / \sqrt{|a|}$, and $\widetilde{C}=C /|a|$, if $P(x)=a x^{2}+b x+c$ and $Q(y)=A y^{2}+B y+C$ with $a A \neq 0$.

In each expression the sign " \pm " corresponds to " + " for $a>0$ and " $-"$ for $a<0$.

In Theorem 1.1 we get that the flow of the Hamiltonian system (3) associated to the rational Hamiltonians $H_{11}^{-}$and $H_{21}^{-}$are topologically equivalent to the one of the Hamiltonians $H_{11}^{+}$and $H_{21}^{+}$, respectively. For instance, the phase portraits of the rational Hamiltonian system of $H_{11}^{-}$can be obtained from $H_{11}^{+}$by simply doing the changes $(x, b) \rightarrow(-x,-b)$. We will classify the global phase portraits of the families of the systems of Theorem 1.1 in the Poincaré disk, and will provide the bifurcation diagrams of these phase portraits.

In order to classify the phase portraits of the polynomial systems (3), the main step is the characterization of the finite and infinite equilibrium points in the Poincaré compactification, for more details about the Poincaré compactification see [9]. The second step for determining the global flow of these polynomial vector fields is to characterize their separatrices. It is known that the separatrices of a polynomial differential system are all the infinite orbits, all the finite singular points, the separatrices of the hyperbolic sectors of the finite and infinite singular points, and the limit cycles. Since the existence of a first integral prevents the existence of limit cycles, we do not have to determine the limit cycles of system 
(3). In the Poincaré disk $\mathbb{D}^{2}$, let $\Sigma$ be the closed set formed by all the separatrices, the components of $\mathbb{D}^{2} \backslash \Sigma$ are called the canonical regions (for more details see [9]). We denote by $\mathrm{S}$ and $\mathrm{R}$ the number of separatrices and canonical regions of a given phase portrait, respectively.

In the phase portraits of the following theorems the straight lines of the set $L$ are indicated by the dash line "- - - ". A small circle in the phase portraits denotes an equilibrium point of the polynomial system (5), but no an equilibrium point of the rational Hamiltonian system (3), and we called it a virtual equilibrium point or a virtual singular point.

Theorem 1.2. The global phase portraits of the rational Hamiltonian system (3) with $H_{11}^{ \pm}=\frac{y^{2}}{2} \pm \frac{x+b}{y+B}$ given in statement (I) of Theorem 1.1 are topologically equivalent to 1.1 of Figure 1.

Theorem 1.3. The global phase portraits of the rational Hamiltonian system (3) with $H_{21}^{ \pm}=\frac{y^{2}}{2} \pm \frac{x^{2}+\Delta}{y+B}$ given in statement (II) of Theorem 1.1 are topologically equivalent to the following ones of Figure 1:

1.2 if $\Delta=B=0$;

1.3 if $\Delta=0$ and $B>0$;

1.4 if $\Delta=0$ and $B<0$;

1.5 if $\Delta>0$ and $4 B^{3}+27 \Delta>0$;

1.6 if $\Delta>0$ and $4 B^{3}+27 \Delta=0$;

1.7 if $\Delta>0$ and $4 B^{3}+27 \Delta<0$;

1.8 if $\Delta<0$ and $4 B^{3}+27 \Delta<0$;

1.9 if $\Delta<0$ and $4 B^{3}+27 \Delta=0$;

1.10 if $\Delta<0$ and $4 B^{3}+27 \Delta>0$.

Moreover the corresponding bifurcation diagram is shown in Figure 4.

Theorem 1.4. The global phase portraits of the rational Hamiltonian system (3) with $H_{12}=\frac{y^{2}}{2}+\frac{x+b}{y^{2}+B y+C}$ given in statement (III) of Theorem 1.1 are topologically equivalent to the following ones of Figure 1:

1.11 if $B^{2}-4 C=0$;

1.12 if $B^{2}-4 C<0$;

1.13 if $B^{2}-4 C>0$.

Moreover the corresponding bifurcation diagram is shown in Figure 5.

Theorem 1.5. The global phase portraits of the rational Hamiltonian system (3) with $H_{22}^{+}=\frac{y^{2}}{2}+\frac{x^{2}+\Delta}{y^{2}+B y+C}$ given in statement (IV) of Theorem 1.1 are topologically equivalent to the following ones of Figures 1 and 2:

1.14 if $B=C=\Delta=0$;

1.15 if $C=B^{2} / 4>0$ and $\Delta=0$;

1.16 if $C=B^{2} / 4$ and $\Delta>0$;

1.17 if $C=B^{2} / 4>0$ and $-27 B^{4} / 8192<\Delta<0$;

1.18 if $C=B^{2} / 4>0$ and $\Delta=-27 B^{4} / 8192$;

1.19 if $C=B^{2} / 4$ and $\Delta<-27 B^{4} / 8192$;

1.20 if $B=0, C<0$ and $0<\Delta<C^{2} / 2$,

or $B \neq 0, C<B^{2} / 4,0<\Delta<F_{4}$ and $D_{5}>0$;

1.21 if $B \neq 0, C<B^{2} / 4,0<\Delta<F_{4}$ and $D_{5}=0$;

1.22 if $B=0, C<0$ and $\Delta \geq C^{2} / 2$, or $B \neq 0, C<B^{2} / 4, \Delta>0$ and $D_{5}<0$; 

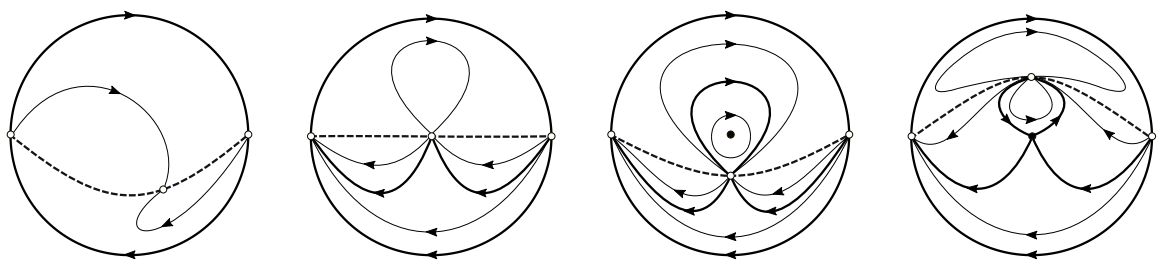

(1.1) $\mathrm{S}=2, \mathrm{R}=2$;

(1.2) $\mathrm{S}=4, \mathrm{R}=4$;

(1.3) $\mathrm{S}=6, \mathrm{R}=5$;
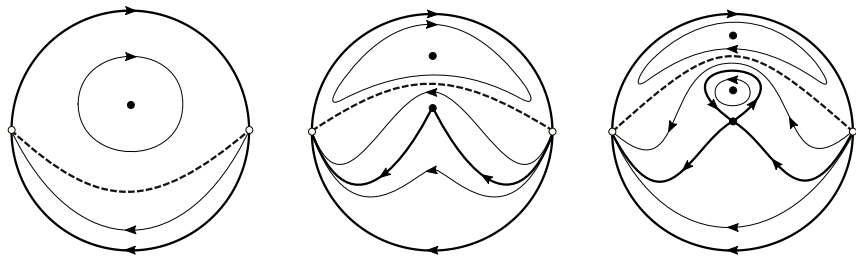

(1.4) $\mathrm{S}=7, \mathrm{R}=5$;

(1.5) $\mathrm{S}=3, \mathrm{R}=2$;

(1.6) $\mathrm{S}=6, \mathrm{R}=3$;

(1.7) $\mathrm{S}=8, \mathrm{R}=4$;
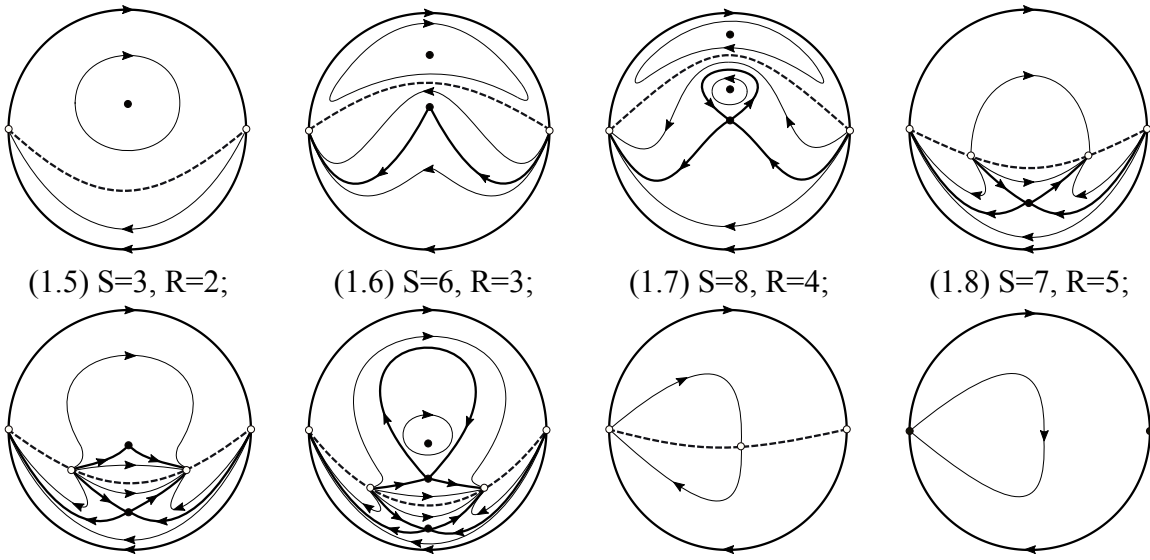

(1.8) $\mathrm{S}=7, \mathrm{R}=5$;

(1.9) $\mathrm{S}=10, \mathrm{R}=6$;

(1.10) $\mathrm{S}=12, \mathrm{R}=7$;

(1.11) $\mathrm{S}=2, \mathrm{R}=2$;
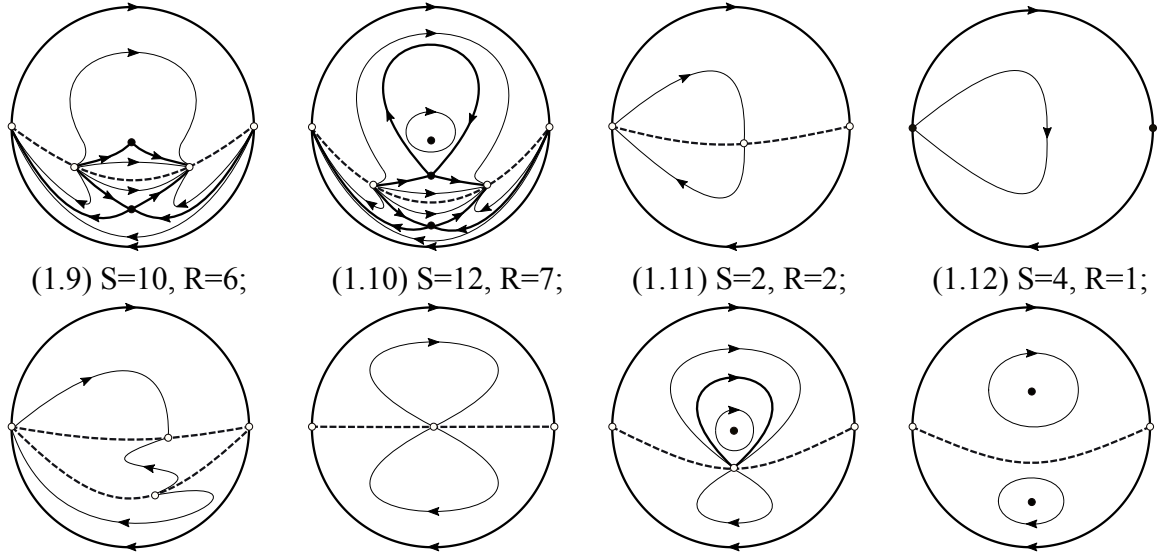

(1.12) $\mathrm{S}=4, \mathrm{R}=1$;

(1.13) $\mathrm{S}=2, \mathrm{R}=3$;

(1.14) $\mathrm{S}=2, \mathrm{R}=2$;

(1.15) $\mathrm{S}=4, \mathrm{R}=3$;
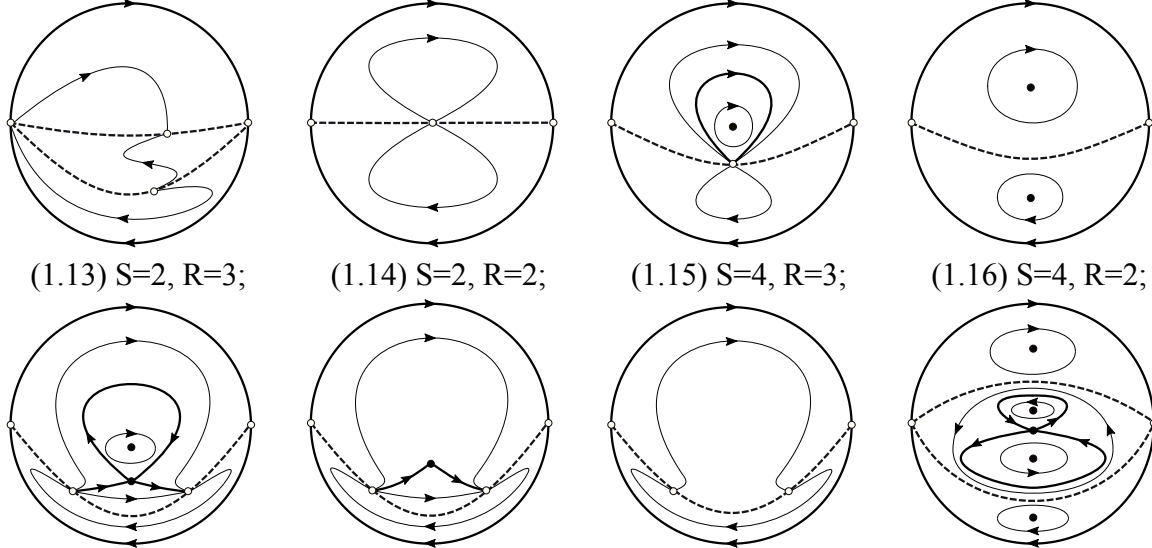

(1.16) $\mathrm{S}=4, \mathrm{R}=2$;

(1.17) $\mathrm{S}=7, \mathrm{R}=4$;

(1.18) $\mathrm{S}=5, \mathrm{R}=3$;

(1.19) $\mathrm{S}=2, \mathrm{R}=2$;
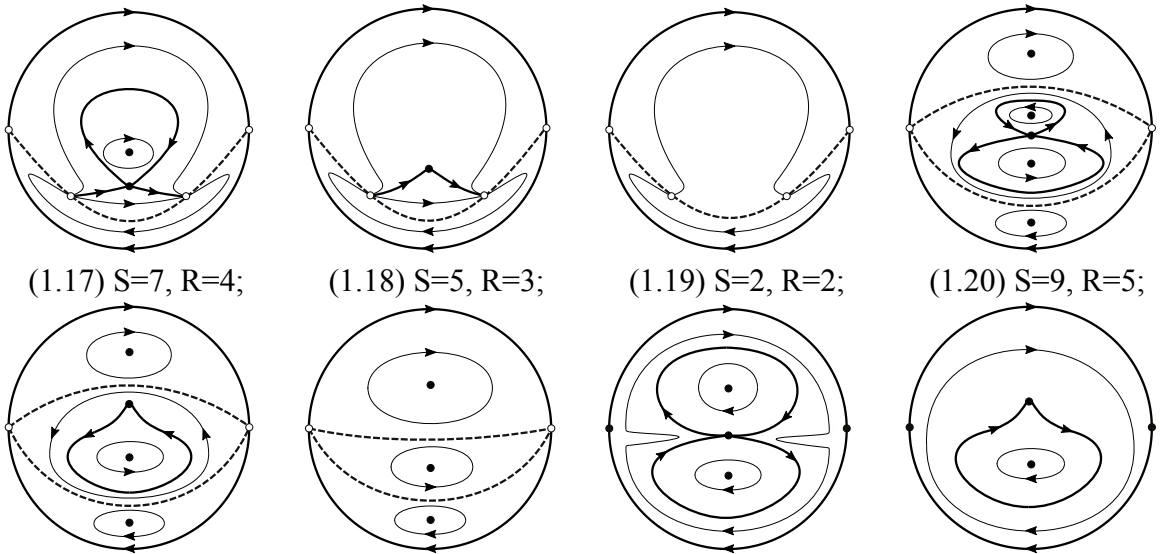

(1.20) $\mathrm{S}=9, \mathrm{R}=5$;

(1.21) $\mathrm{S}=7, \mathrm{R}=4$;

(1.22) $\mathrm{S}=5, \mathrm{R}=3$;

(1.23) $\mathrm{S}=9, \mathrm{R}=3$;

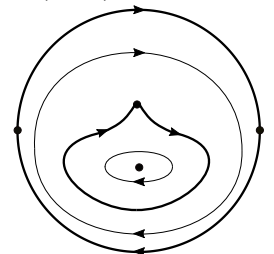

(1.24) $\mathrm{S}=7, \mathrm{R}=2$.

Figure 1. Topological phase portraits of cases 1.1-1.24 in Theorems 1.2-1.5. 

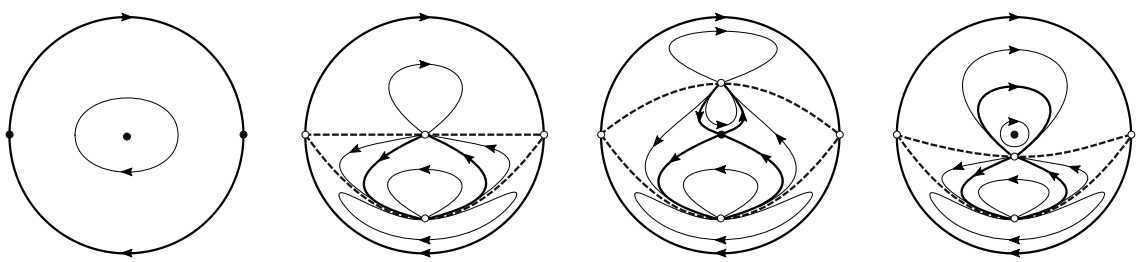

(1.25) $\mathrm{S}=5, \mathrm{R}=1$;

(1.26) $\mathrm{S}=4, \mathrm{R}=5$;

(1.27) $\mathrm{S}=7, \mathrm{R}=6$;

(1.28) $\mathrm{S}=6, \mathrm{R}=6$;
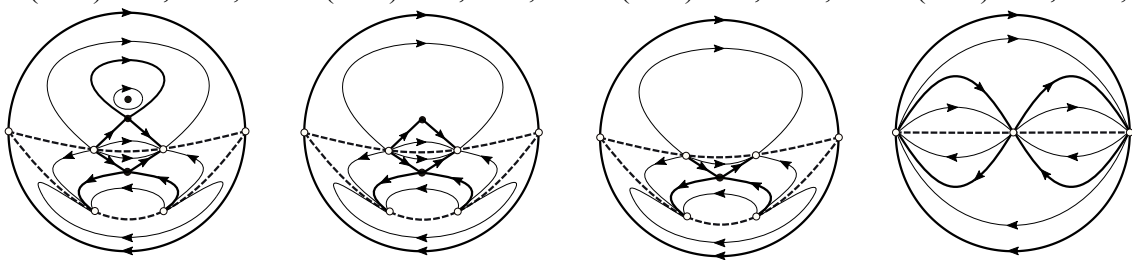

(1.29) $\mathrm{S}=12, \mathrm{R}=8$;

(1.30) $\mathrm{S}=10, \mathrm{R}=7$;

(1.31) $\mathrm{S}=7, \mathrm{R}=6$;

(1.32) $\mathrm{S}=6, \mathrm{R}=6$;
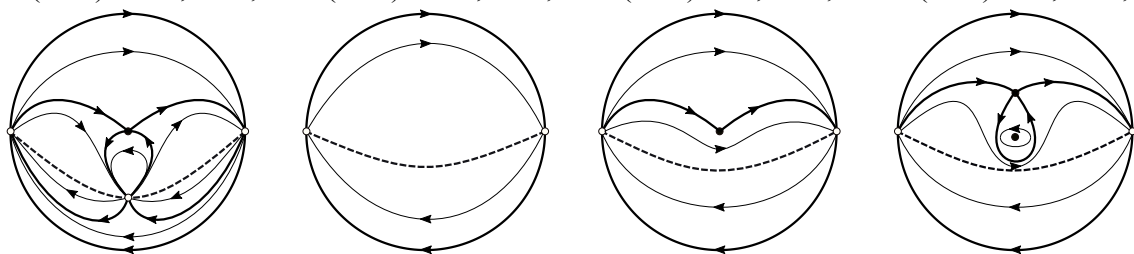

(1.33) $\mathrm{S}=9, \mathrm{R}=7$;

(1.34) $\mathrm{S}=2, \mathrm{R}=2$;

(1.35) $\mathrm{S}=5, \mathrm{R}=3$;

(1.36) $\mathrm{S}=7, \mathrm{R}=4$;
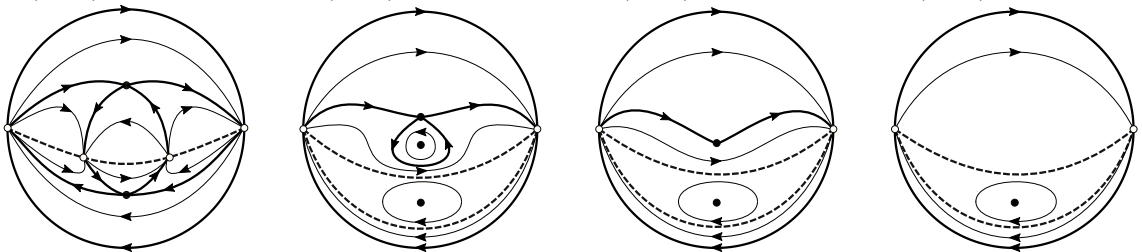

(1.37) $\mathrm{S}=12, \mathrm{R}=8$;

(1.38) $\mathrm{S}=8, \mathrm{R}=5$;

(1.39) $\mathrm{S}=6, \mathrm{R}=4$;

(1.40) $\mathrm{S}=3, \mathrm{R}=3$;
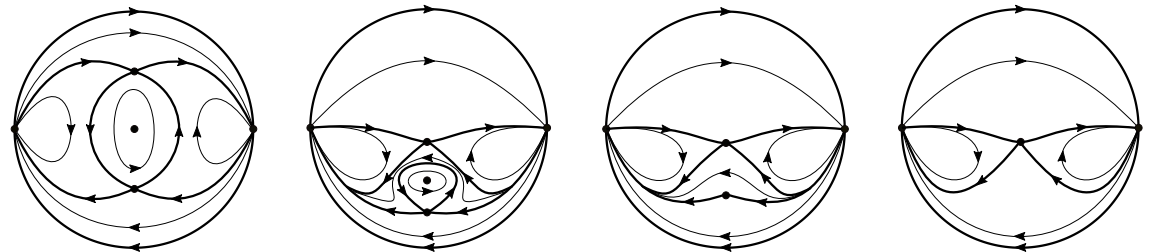

(1.41) $\mathrm{S}=13, \mathrm{R}=5$;

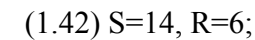

(1.43) $\mathrm{S}=12, \mathrm{R}=5$;
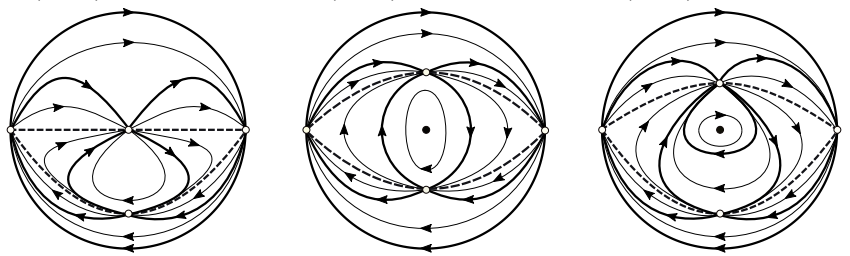

(1.44) $\mathrm{S}=9, \mathrm{R}=4$;

(1.45) $\mathrm{S}=8, \mathrm{R}=9$;

(1.46) $\mathrm{S}=9, \mathrm{R}=9$;

(1.47) $\mathrm{S}=10, \mathrm{R}=10$;

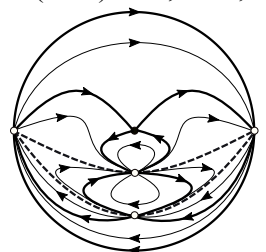

(1.48) $\mathrm{S}=11, \mathrm{R}=10$.

Figure 2. Topological phase portraits of cases 1.25-1.48 in Theorems 1.5 and 1.6. 


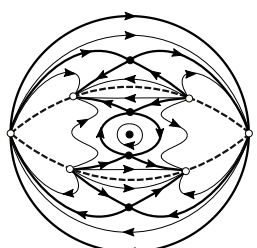

(1.49) $\mathrm{S}=21, \mathrm{R}=13$;

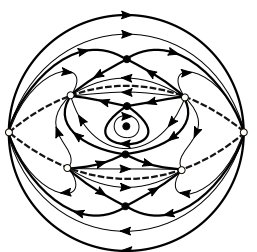

(1.50) $\mathrm{S}=22, \mathrm{R}=14$;

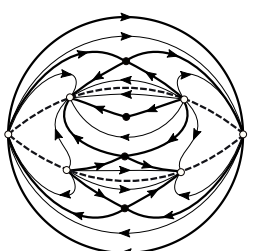

(1.51) $\mathrm{S}=20, \mathrm{R}=13$;

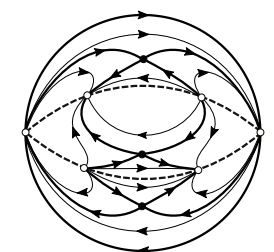

(1.52) $\mathrm{S}=17, \mathrm{R}=12$

Figure 3. Topological phase portraits of cases 1.49-1.52 in Theorem 1.6.

1.23 if $B=0, C>0$ and $\Delta>C^{2} / 2$, or $C>B^{2} / 4>0, \Delta>F_{4}$ and $D_{5}<0$, or $C>B^{2} / 4>0, F_{6}<\Delta<0$ and $D_{5}<0$;

1.24 if $C>B^{2} / 4>0, \Delta>F_{4}$ and $D_{5}=0$, or $C>B^{2} / 4>0, F_{6}<\Delta<0$ and $D_{5}=0$

1.25 if $B=0, C>0$ and $\Delta \leq C^{2} / 2$, or $C>B^{2} / 4>0$ and $\Delta=0$, or $C>B^{2} / 4>0, \Delta \neq 0$ and $D_{5}>0$;

1.26 if $B \neq 0$ and $\Delta=C=0$;

1.27 if $C<0$ and $\Delta=0$;

1.28 if $0<C<B^{2} / 4$ and $\Delta=0$;

1.29 if $0<C<B^{2} / 4, F_{6}<\Delta<0$ and $D_{5}<0$;

1.30 if $0<C<B^{2} / 4, F_{6}<\Delta<0$ and $D_{5}=0$;

1.31 if $B=0, C<0$ and $\Delta<0$, or $B \neq 0, C<B^{2} / 4$ and $\Delta<F_{6}$, or $B \neq 0, C<B^{2} / 4, C \neq 0, F_{6} \leq \Delta<0$ and $D_{5}>0$.

See (63), (64) and (69) for the expression of $D_{5}, F_{4}$ and $F_{6}$, respectively. Moreover the corresponding bifurcation diagrams are shown in Figures 6-8.

Theorem 1.6. The global phase portraits of the rational Hamiltonian system (3) with $H_{22}^{-}=\frac{y^{2}}{2}-\frac{x^{2}+\Delta}{y^{2}+B y+C}$ given in statement $(I V)$ of Theorem 1.1 are topologically equivalent to the following ones of Figures 2 and 3:

1.32 if $B=C=\Delta=0$;

1.33 if $C=B^{2} / 4>0$ and $\Delta=0$;

1.34 if $C=B^{2} / 4$ and $\Delta>27 B^{4} / 8192$;

1.35 if $C=B^{2} / 4>0$ and $\Delta=27 B^{4} / 8192$;

1.36 if $C=B^{2} / 4>0$ and $0<\Delta<27 B^{4} / 8192$;

1.37 if $C=B^{2} / 4$ and $\Delta<0$;

1.38 if $B \neq 0, C<B^{2} / 4, C \neq 0,0<\Delta<-F_{6}$ and $D_{5}(-\Delta, B, C)<0$;

1.39 if $B \neq 0, C<B^{2} / 4, C \neq 0,0<\Delta<-F_{6}$ and $D_{5}(-\Delta, B, C)=0$;

1.40 if $B=0, C<0$, and $\Delta>0$, or $B \neq 0, C<B^{2} / 4, C \neq 0, \Delta>0$ and $D_{5}(-\Delta, B, C)>0$;

1.41 if $B=0, C>0$, and $\Delta<-C^{2} / 2$;

1.42 if $C>B^{2} / 4>0, \Delta<-F_{4}$ and $D_{5}(-\Delta, B, C)<0$, or $C>B^{2} / 4>0,0<\Delta<-F_{6}$ and $D_{5}(-\Delta, B, C)<0$;

1.43 if $C>B^{2} / 4>0,0<\Delta<-F_{6}$ and $D_{5}(-\Delta, B, C)=0$, or $C>B^{2} / 4>0, \Delta<-F_{4}$ and $D_{5}(-\Delta, B, C)=0$;

1.44 if $B=0, C>0$ and $\Delta \geq-C^{2} / 2$, or $C>B^{2} / 4>0$ and $\Delta=0$, or $C>B^{2} / 4>0, \Delta \neq 0$ and $D_{5}(-\Delta, B, C)>0$;

1.45 if $B \neq 0$ and $C=\Delta=0$;

1.46 if $C<0$ and $B=\Delta=0$; 
1.47 if $B \neq 0, C<0$ and $\Delta=0$;

1.48 if $0<C<B^{2} / 4$ and $\Delta=0$;

1.49 if $B=0, C<0$ and $-C^{2} / 2<\Delta<0$;

1.50 if $B \neq 0, C<B^{2} / 4,-F_{4}<\Delta<0$ and $D_{5}(-\Delta, B, C)>0$;

1.51 if $B \neq 0, C<B^{2} / 4,-F_{4}<\Delta<0$ and $D_{5}(-\Delta, B, C)=0$;

1.52 if $B=0, C<0$ and $\Delta \leq-C^{2} / 2$,

or $B \neq 0, C<B^{2} / 4, \Delta<0$ and $D_{5}(-\Delta, B, C)<0$.

See (63), (64) and (69) for the expression of $D_{5}, F_{4}$ and $F_{6}$, respectively. Moreover the corresponding bifurcation diagrams are shown in Figures 9-11.

We will give some preliminary definitions and theorems in the following section. We prove how to obtain the normal forms given in Theorem 1.1 in Section 3. We determine the phase portraits of the vector fields (I)-(IV) in Sections 4-7, respectively, in other words we prove Theorems $1.2-1.5$. In a similar way to the proof of Theorem 1.5 we can obtain the phase portraits of the Hamiltonian $\mathrm{H}_{22}^{-}$, but for the sake of simplicity we will not provide the proof of Theorem 1.6 here.

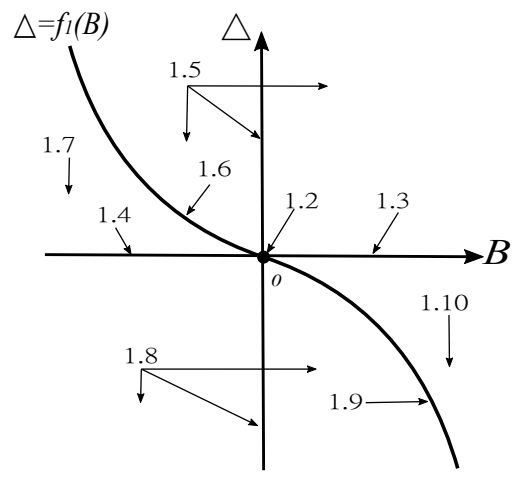

FiguRE 4. The bifurcation diagram of the phase portraits for $H_{21}^{ \pm}=\frac{y^{2}}{2} \pm \frac{x^{2}+\Delta}{y+B}$ where $f_{1}(B)=-4 B^{3} / 27$.

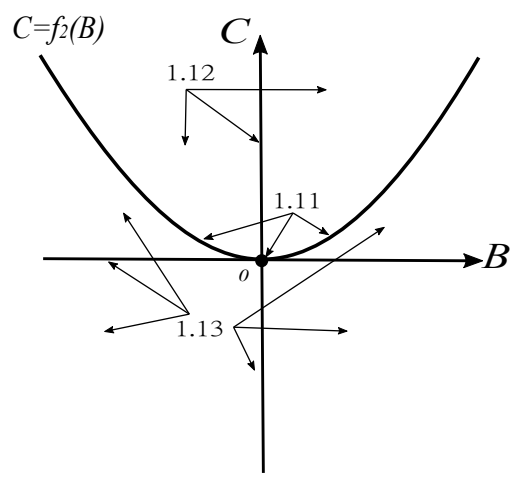

Figure 5. The bifurcation diagram of the phase portraits for $H_{12}=\frac{y^{2}}{2}+\frac{x+b}{y^{2}+B y+C}$ where $f_{2}(B)=B^{2} / 4$. 


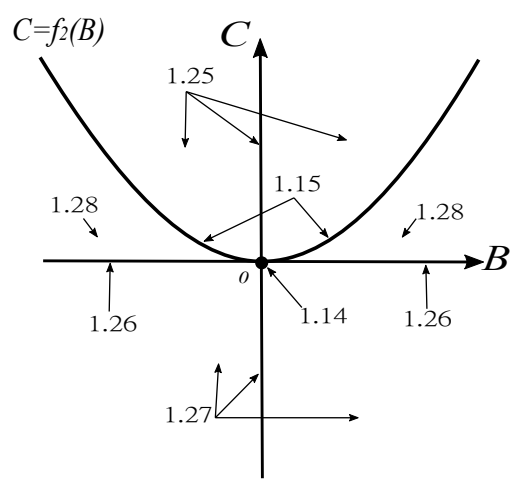

Figure 6. The bifurcation diagram of the phase portraits for $H_{22}^{+}=\frac{y^{2}}{2}+\frac{x^{2}+\Delta}{y^{2}+B y+C}$ with $\Delta=0$, where $f_{2}(B)=B^{2} / 4$.

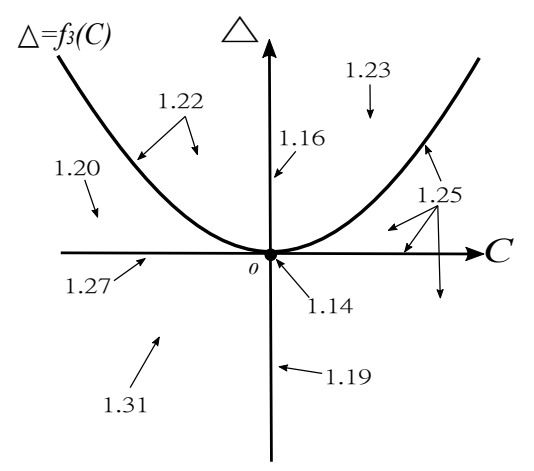

Figure 7. The bifurcation diagram of the phase portraits for $H_{22}^{+}=\frac{y^{2}}{2}+\frac{x^{2}+\Delta}{y^{2}+B y+C}$ with $B=0$, where $f_{3}(C)=C^{2} / 2$.

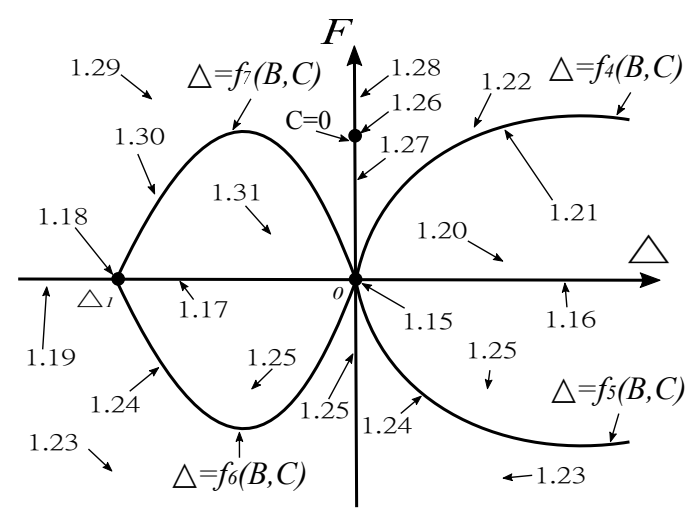

Figure 8. The bifurcation diagram of the phase portraits for $H_{22}^{+}=\frac{y^{2}}{2}+\frac{x^{2}+\Delta}{y^{2}+B y+C}$ with $B \neq 0$, where $F=B^{2}-4 C$, $\Delta_{1}=-27 B^{4} / 8192$ and $f_{4,5,6,7}(B, C)$ are convenient functions. 


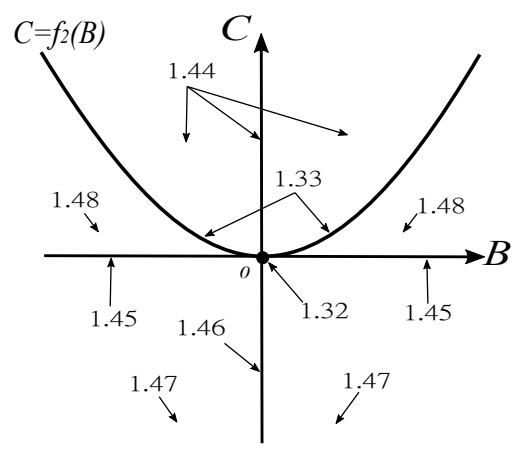

Figure 9. The bifurcation diagram of the phase portraits for $H_{22}^{-}=\frac{y^{2}}{2}-\frac{x^{2}+\Delta}{y^{2}+B y+C}$ with $\Delta=0$, where $f_{2}(B)=B^{2} / 4$.

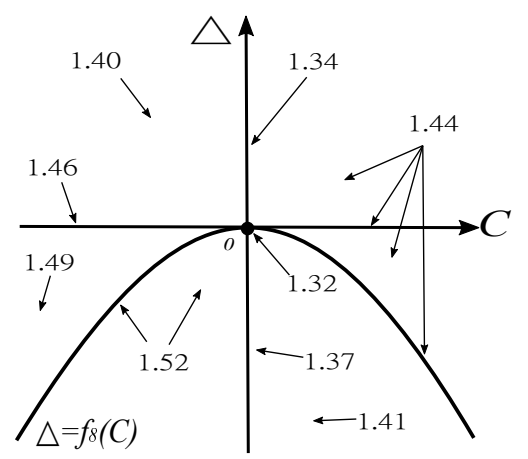

Figure 10. The bifurcation diagram of the phase portraits for $H_{22}^{-}=\frac{y^{2}}{2}-\frac{x^{2}+\Delta}{y^{2}+B y+C}$ with $B=0$, where $f_{8}(C)=-C^{2} / 2$.

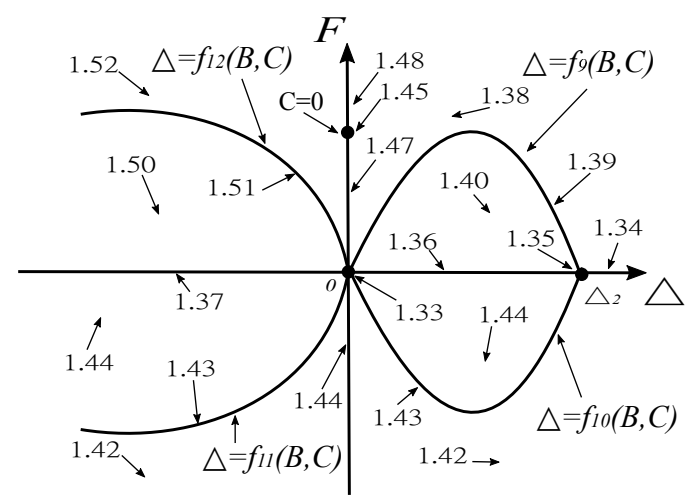

Figure 11. The bifurcation diagram of the phase portraits for $H_{22}^{-}=\frac{y^{2}}{2}-\frac{x^{2}+\Delta}{y^{2}+B y+C}$ with $B \neq 0$, where $F=B^{2}-4 C, \Delta_{2}=$ $27 B^{4} / 8192$ and $f_{9,10,11,12}(B, C)$ are convenient functions.

\section{Preliminaries}

Now we introduce some basic results that we will need for the analysis of the local phase portraits of the finite and infinite singular points of the polynomial vector fields. 
2.1. Poincaré compactification. In this subsection we present some preliminaries about the Poincaré disk and the compactification of a polynomial vector field, for more details see Chapter 5 of [9].

We denote by $P\left(\mathbb{R}^{2}\right)$ the set of all polynomial vector fields on $\mathbb{R}^{2}$ of the form

$$
X=\left(\dot{x_{1}}, \dot{x_{2}}\right)=\left(X\left(x_{1}, x_{2}\right), Y\left(x_{1}, x_{2}\right)\right),
$$

where $X\left(x_{1}, x_{2}\right)$ and $Y\left(x_{1}, x_{2}\right)$ are real polynomials in the variables $x_{1}$ and $x_{2}$ of degree $d$. We consider $\mathbb{R}^{2}$ as the plane in $\mathbb{R}^{3}$ defined by $\mathbf{s}=\left(s_{1}, s_{2}, s_{3}\right)=\left(x_{1}, x_{2}, 1\right)$. The plane $\mathbb{R}^{2}$ is tangent to the Poincaré sphere $\mathbb{S}^{2}=\left\{\mathbf{s} \in \mathbb{R}^{3}:\|\mathbf{s}\|=1\right\}$ at the north pole. We consider the central projection of $\mathbb{R}^{2}$ onto the sphere $\mathbb{S}^{2}$, that is, for each point $x \in \mathbb{R}^{2}=\left(x_{1}, x_{2}, 1\right) \in \mathbb{R}^{3}$ we take the straight line $l$ through this point and the origin of $\mathbb{R}^{3}$ and the central projection sends the point $x$ to the two intersection points of the straight line $l$ with the sphere $\mathbb{S}^{2}$. Then we obtain a vector field $X^{\prime}$ formed by two copies of $X$ : one on the northern hemisphere $\left\{\mathbf{s} \in \mathbb{S}^{2}: s_{3}>0\right\}$, and the other on the southern hemisphere $\left\{\mathbf{s} \in \mathbb{S}^{2}: s_{3}<0\right\}$. Note that the equator $\mathbb{S}^{1}=\left\{\mathbf{s} \in \mathbb{S}^{2}: s_{3}=0\right\}$ corresponds to the infinity of $\mathbb{R}^{2}$. This vector field $X^{\prime}$ on $\mathbb{S}^{2} \backslash \mathbb{S}^{1}$ can be extended to a vector field $p(X)$ on the whole sphere $\mathbb{S}^{2}$ multiplying the vector field $X^{\prime}$ by $x_{3}^{d}$. The vector field $p(X)$ is called the Poincaré compactification of the vector field $X$.

In order to compute the expression of the vector field $p(X)$, we consider the six local charts given by

$$
U_{i}=\left\{\mathbf{s} \in \mathbb{S}^{2}: s_{i}>0\right\}, \quad V_{i}=\left\{\mathbf{s} \in \mathbb{S}^{2}: s_{i}<0\right\},
$$

for $i=1,2,3$, and the diffeomorphisms

$$
\varphi_{i}(\mathbf{s}): U_{i} \rightarrow \mathbb{R}^{2}, \quad \psi_{i}(\mathbf{s}): V_{i} \rightarrow \mathbb{R}^{2},
$$

defined as $\varphi_{i}(\mathbf{s})=-\psi_{i}(\mathbf{s})=\left(s_{m} / s_{i}, s_{n} / s_{i}\right)=(u, v)$ for $m<n$ and $m, n \neq i$. We note that $(u, v)$ will play different roles in every local chart. Then the expression of $p(X)$ in the local chart $U_{1}$ is

$$
\dot{u}=v^{d}\left[-u X\left(\frac{1}{v}, \frac{u}{v}\right)+Y\left(\frac{1}{v}, \frac{u}{v}\right)\right], \quad \dot{v}=-v^{d+1} X\left(\frac{1}{v}, \frac{u}{v}\right) .
$$

Analogously, the expression of $p(X)$ in the local chart $U_{2}$ is

$$
\dot{u}=v^{d}\left[-u X\left(\frac{u}{v}, \frac{1}{v}\right)-u Y\left(\frac{u}{v}, \frac{1}{v}\right)\right], \quad \dot{v}=-v^{d+1} Y\left(\frac{u}{v}, \frac{1}{v}\right),
$$

and in $U_{3}$ is

$$
\dot{u}=X(u, v), \dot{v}=Y(u, v) .
$$

The expression of $p(X)$ in the local charts $V_{i}$ is the same as in $U_{i}$ multiplied by $(-1)^{d-1}$ for $i=1,2,3$. That is, if $\mathbf{s}$ is an equilibrium point, then $-\mathbf{s}$ is also an equilibrium point. Note that the local behavior near $-\mathbf{s}$ is the local behavior near $\mathbf{s}$ multiplied by $(-1)^{d-1}$. Hence it is enough to study the Poincaré compactification restricted to the northern hemisphere plus $\mathbb{S}^{1}$ to study the vector field $X$. For drawing the phase portraits we will consider the orthogonal projection $\pi(\mathbf{s})=$ $\left(s_{1}, s_{2}\right)$ of the northern hemisphere onto the closed unit disk $\mathbb{D}^{2}$ centered at the origin of coordinates in the plane $s_{3}=0 . \mathbb{D}^{2}$ is called the Poincaré disk. Infinite equilibrium points of $X$ are the equilibrium points of the vector field $p(X)$ which are on $\mathbb{S}^{1}$. So for studying the infinite equilibrium points it suffices to look the ones 
at $\left.U_{1}\right|_{v=0}$ and at the origin of $U_{2}$. We compute the finite equilibrium points of $X$ by the chart $U_{3}$, which are the equilibrium points of $p(X)$ in $\mathbb{S}^{2} \backslash \mathbb{S}^{1}$.

2.2. Some other preliminaries. For proving our main result we need to study different types of equilibria, where the linearly zero equilibria are studied using the changes of variables called blow-up's. For more details about the blow-up's see [1].

Sometime the explicit expressions of the finite equilibrium points and their eigenvalues in terms of the parameters are complicated, therefore it is hard to analyze their existence and their local phase portraits. For this reason we will provide an alternative way for studying the finite equilibria. Firstly we present one way to determine the real roots of the polynomial

$$
f(x)=a_{0} x^{n}+a_{1} x^{n-1}+\cdots+a_{n} .
$$

We compute the discriminant sequence $\left\{D_{1}, D_{2}, \cdots, D_{n}\right\}$ of the polynomial (12) (see more details in [17]) and determine the sign list

$$
\left[\operatorname{sign}\left(D_{1}\right), \operatorname{sign}\left(D_{2}\right), \cdots, \operatorname{sign}\left(D_{n}\right)\right]
$$

of the discriminant sequence, where the sign function is

$$
\operatorname{sign}(x)=\left\{\begin{aligned}
1, & \text { if } x>0, \\
0, & \text { if } x=0, \\
-1, & \text { if } x<0 .
\end{aligned}\right.
$$

Then we construct the associated revised sign list $\left[r_{1}, r_{2}, \cdots, r_{n}\right]$ which will give all the information about the number of real roots of the polynomial (12). For any sign list $\left[s_{1}, s_{2}, \cdots, s_{n}\right]$, the revised sign list $\left[r_{1}, r_{2}, \cdots, r_{n}\right]$ is obtained as follows:

1. If $s_{k} \neq 0$ we write $r_{k}=s_{k}$.

2. If $\left[s_{i}, s_{i+1}, \cdots, s_{i+j}\right]$ is a section of the given sign list such that $s_{i+1}=\cdots=$ $s_{i+j-1}=0$ with $s_{i} s_{i+j} \neq 0$, we replace the subsection $\left[s_{i+1}, s_{i+2}, \cdots, s_{i+j-1}\right]$ with $\left[-s_{i},-s_{i}, s_{i}, s_{i},-s_{i},-s_{i}, s_{i}, s_{i},-s_{i}, \cdots\right]$ keeping the number of terms.

As a result there are no zeros between nonzero elements of the revised sign list. Thus for instance the revision of the sign list $[1,-1,0,0,0,0,0,1,-1,0,0]$ is

$$
[1,-1,1,1,-1,-1,1,1,-1,0,0] \text {. }
$$

From [17] we have the following theorem.

Theorem 2.1. For a polynomial (12) if the number of the sign changes of the revised sign list of $\left[r_{1}, r_{2}, \cdots, r_{n}\right]$ is $v$, and the number of nonzero members of the revised sign list is $l$, then the number of the distinct real roots of (12) equals $l-2 v$.

When we find the number of finite equilibrium points of a system, we can compute the topological indices of the equilibrium points, both finite and infinite. Here we will present two important theorems, the Poincaré Formula and the PoincaréHopf Theorem. The former allows to compute the index of an equilibrium point of a planar vector field. The latter is suitable for the systems in a 2-dimensional sphere. For more details about these theorems see Chapter 6 of [9]. 
Theorem 2.2 (Poincaré Formula). Let $q$ be an isolated equilibrium point having the finite sectorial decomposition property. Let $e, h$ and $p$ denote the number of elliptic, hyperbolic and parabolic sectors of $q$, respectively. Then the index of $q$ is $(e-h) / 2+1$.

Corollary 2.3. The index of a node, a center, a saddle and a cusp are 1, 1, -1 and 0 , respectively.

Theorem 2.4 (Poincaré-Hopf Theorem). For every vector field on the sphere $\mathbb{S}^{2}$ with a finite number of equilibrium points, the sum of the indices of all its equilibria is 2 .

We have the following remark.

Remark 2.5. Since the flow of Hamiltonian systems preserves the area, we have that any finite equilibrium of Hamiltonian systems must be either a center, or a union of an even number of hyperbolic sectors. In particular, the finite nilpotent equilibrium points of Hamiltonian planar polynomial vector fields are either saddles, centers, or cusps, for more details see Theorem 3.5 of [9].

\section{Proof of Theorem 1.1}

(I) If $P(x)=a x+b, Q(y)=A y+B$ and $a A \neq 0$, we have $V(x, y)=\frac{a x / A+b / A}{y+B / A}$. Without loss of generality we can take $A=1$, and obtain the rational potential $V(x, y)=\frac{a x+b}{y+B}$. By the linear change of variables

$$
X=\frac{x}{\sqrt{|a|}}, \quad Y=\frac{y}{\sqrt{|a|}}
$$

the Hamiltonian function (2) will be the form $H_{11}^{ \pm}=\frac{Y^{2}}{2} \pm \frac{X+b /(a \sqrt{|a|})}{Y+B / \sqrt{|a|}}$.

(II) In the case $V(x, y)=\frac{a x^{2}+b x+c}{A y+B}$ with $a A \neq 0$, without loss of generality we can take $A=1$, and have $V(x, y)=\frac{a(x+b /(2 a))^{2}+c-b^{2} /(4 a)}{y+B}$. Considering the linear change of variables

$$
X=\sqrt{|a|}\left(x+\frac{b}{2 a}\right), \quad Y=y,
$$

and writing $\Delta=c-b^{2} /(4 a)$, the Hamiltonian function (2) has the form $H_{21}^{ \pm}=$ $\sqrt{|a|}\left(\frac{Y^{2}}{2} \pm \frac{X^{2} \pm \Delta}{Y+B}\right)$. Doing the rescaling in time $d t=d \tau / \sqrt{|a|}$ we get the normalized Hamiltonian function $H_{21}^{ \pm}=\frac{Y^{2}}{2} \pm \frac{X^{2} \pm \Delta}{Y+B}$.

(III) For $V(x, y)=\frac{a x+b}{A y^{2}+B y+C}$ with $a A \neq 0$, without loss of generality we can take $A=1$, and consequently $V(x, y)=\frac{a y+b}{y^{2}+B y+C}$. Using a suitable change of variables

$$
X=\frac{x}{\sqrt[3]{a}}, \quad Y=\frac{y}{\sqrt[3]{a}}
$$

we have the normalized Hamiltonian function $H_{12}=\frac{Y^{2}}{2}+\frac{X+b /(a \sqrt[3]{a})}{Y^{2}+B Y / \sqrt[3]{a}+C / \sqrt[3]{a^{2}}}$. 
(IV) Finally in the case $V(x, y)=\frac{a x^{2}+b x+c}{A y^{2}+B y+C}$, without loss of generality we can take $A=1$, and so $V(x, y)=\frac{a(x+b /(2 a))^{2}+c-b^{2} /(4 a)}{y^{2}+B y+C}$. Doing the change of variables

$$
X=\frac{x+b /(2 a)}{\sqrt{|a|}}, \quad Y=\frac{y}{\sqrt{|a|}},
$$

the normalized Hamiltonian function (2) will be $H_{22}^{ \pm}=\frac{Y^{2}}{2} \pm \frac{X^{2}+\Delta}{Y^{2}+B Y / \sqrt{|a|}+C /|a|}$ where $\Delta=\left(c / a-b^{2} /\left(4 a^{2}\right)\right) /|a|$. This completes the proof of Theorem 1.1.

\section{Proof of Theorem 1.2}

The rational Hamiltonian function given in statement (I) of Theorem 1.1 is

$$
H_{11}^{ \pm}(x, y)=\frac{y^{2}}{2} \pm \frac{x+b}{y+B},
$$

and the associated Hamiltonian system for $H_{11}^{+}$is

$$
\dot{x}=y-\frac{x+b}{(y+B)^{2}}, \quad \dot{y}=-\frac{1}{y+B} .
$$

Note that we can assume $B \geq 0$ because system (14) is invariant under the transformation $(x, y, t, b, B) \rightarrow(-x,-y, t,-b,-B)$.

We apply the rescaling in time $d t=(y+B)^{2} d \tau$, and system (14) becomes

$$
x^{\prime}=-x-b+y(y+B)^{2}, \quad y^{\prime}=-(y+B) .
$$

We study the dynamics of the infinite equilibrium points of system (15) through the Poincaré compactification. In the local chart $U_{1}$ system (15) becomes

$$
\begin{aligned}
& u^{\prime}=-u^{4}-2 B u^{3} v-B^{2} u^{2} v^{2}-B v^{3}+b u v^{3}, \\
& v^{\prime}=v\left(v^{2}+b v^{3}-u^{3}-2 B u^{2} v-B^{2} u v^{2}\right) .
\end{aligned}
$$

On the infinity, i.e. on $v=0$, the origin is the unique equilibrium point, and its the linear part is identically zero. In order to describe the local phase portrait at the origin of $U_{1}$ we do the blow-up $(u, v) \rightarrow(u, w)$ with $w=v / u$. Then we have the system

$$
\begin{aligned}
u^{\prime} & =-u^{3}\left(u+2 B u w+B^{2} u w^{2}+B w^{3}-b u w^{3}\right), \\
w^{\prime} & =u^{2} w^{3}(1+B w) .
\end{aligned}
$$

Doing a rescaling of the time we eliminate the common factor $u^{2}$ between $u^{\prime}$ and $w^{\prime}$. Then we obtain the system

$$
\begin{aligned}
u^{\prime} & =-u\left(u+2 B u w+B^{2} u w^{2}+B w^{3}-b u w^{3}\right), \\
w^{\prime} & =w^{3}(1+B w) .
\end{aligned}
$$

When $u=0$ system (18) can have two equilibrium points $E_{1}=(0,0)$ and $E_{2}=$ $(0,-1 / B)$ if $B \neq 0$.

If $B=0$ system (18) has only the equilibrium point $E_{1}$ on $u=0$, which is linearly zero. We do a second directional blow-up $(u, w) \rightarrow(u, \widetilde{w})$ with $\widetilde{w}=w / u$ and removing the common factor $u$, then we obtain the system

$$
u^{\prime}=-u\left(1-b u^{3} \widetilde{w}^{3}\right), \quad \widetilde{w}^{\prime}=\widetilde{w}\left(1+u \widetilde{w}^{2}-b u^{3} \widetilde{w}^{3}\right) .
$$




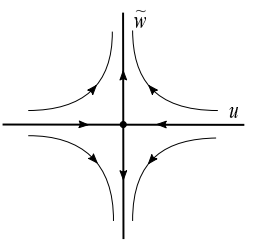

(a)

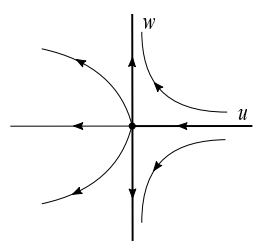

(b)

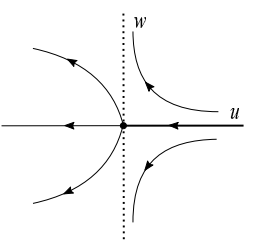

(c)

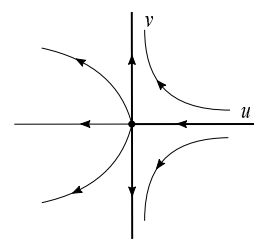

(d)

Figure 12. Blow-up at the origin of system (16) in $U_{1}$ with $B=0$. (a) System (19), (b) System (18), (c) System (17), (d) System (16).

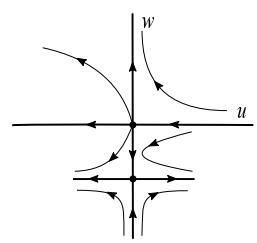

(a)

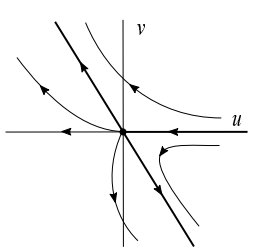

(b)

Figure 13. Blow-up at the origin of system (16) in $U_{1}$ with $B>0$.

(a) System (18), (b) System (16).

When $u=0$ the origin is the unique equilibrium point of system (19), and it is a saddle, with the stable and unstable separatrices on the $u$-axis and $\widetilde{w}$-axis, respectively (see Figure 12(a)). Going back through the change of variables, taking into account that both axes are invariant by system (16), we obtain $\left.u^{\prime}\right|_{v=0}=-u^{4}$ and $\left.v^{\prime}\right|_{u=0}=v^{3}+O\left(v^{4}\right)$. Hence we have that the phase portrait in a neighborhood of the origin of system (16) consists of two hyperbolic sectors and one parabolic sector (see Figure 12(d)).

If $B>0$ the equilibrium $E_{1}$ is linearly zero and $E_{2}$ is a saddle. The local phase portrait of $E_{1}$ coincides with the one of the case $B=0$, see Figure $12(\mathrm{~b})$. We superpose the study of the equilibria $E_{1,2}$ and obtain the local behavior of system (18), see Figure 13(a). Going back through the change of variables and taking into account the behavior of the flow of system (16) on the axes, we have $\left.u^{\prime}\right|_{u=0}=-B v^{3}$, $\left.u^{\prime}\right|_{v=0}=-u^{4}$ and $\left.v^{\prime}\right|_{u=0}=v^{3}+O\left(v^{4}\right)$. Then we obtain that the origin of system (16) has two hyperbolic and one parabolic sector, see Figure 13(b).

Next we check if the origin of the local chart $U_{2}$ is an equilibrium point, and we get

$$
u^{\prime}=1+2 B v+B^{2} v^{2}-b v^{3}+B u v^{3}, \quad v^{\prime}=v^{3}(1+B v) .
$$

Obviously, the origin of system (20) is not an equilibrium point.

System (15) has two equilibria in the infinite region, one in $U_{1}$, and its diametrically opposite in $V_{1}$. The flow in the chart $V_{1}$ has the same sense as in $U_{1}$ because the degree of system (15) is 3 . Now we must analyze which of the equilibrium points of system (15) in the Poincaré disk are or not effectively equilibrium points of the rational system (14). We observe that the set $L=\{(x, y) \mid y+B=0\}$ corresponds 
to the straight line, which connects the origins in $U_{1}$ and $V_{1}$. Therefore there exist no equilibria at infinity for the rational Hamiltonian system (14).

Having determined the infinite equilibria of system (15) and of the Hamiltonian system (14), we now analyze their finite equilibrium points. The linear part of system (15) is

$$
M=\left(\begin{array}{cc}
-1 & 3 y^{2}+4 B y+B^{2} \\
0 & -1
\end{array}\right)
$$

System (15) has the unique finite equilibrium point $p_{1}=(-b,-B)$. From $(21)$ we get that $p_{1}$ is a hyperbolic attracting node of system (15). In addition, the equilibrium point $p_{1}$ is on the straight line $y=-B$, i.e. in the set $L$. Hence it is a virtual equilibrium point, and system (14) has no finite equilibrium points. Using the level of energy on the $y$-axis $\left.H_{11}^{+}\right|_{x=0}=y^{2} / 2+b /(y+B)=h$ it follows that the integral curves cross the $y$-axis at most twice. Then the global phase portrait of the rational Hamiltonian system (14) is given by 1.1 of Figure 1. This concludes the proof of Theorem 1.2.

\section{Proof of Theorem 1.3}

Next we study the rational Hamiltonian system $H_{21}^{+}=\frac{y^{2}}{2}+\frac{x^{2}+\Delta}{y+B}$, here the Hamiltonian system of $H_{21}^{+}$is

$$
\dot{x}=y-\frac{x^{2}+\Delta}{(y+B)^{2}}, \quad \dot{y}=-\frac{2 x}{y+B} .
$$

We apply the rescaling $d t=(y+B)^{2} d \tau$, and have the system

$$
x^{\prime}=-\left(x^{2}+\Delta\right)+y(y+B)^{2}, \quad y^{\prime}=-2 x(y+B),
$$

which is a polynomial system of degree 3 .

We analyze the infinity of system (23) through the Poincaré compactification. The associated system $(23)$ in $U_{1}$ is

$$
\begin{aligned}
& u^{\prime}=-u^{4}-u v-2 B u^{3} v-2 B v^{2}-B^{2} u^{2} v^{2}+\Delta u v^{3}, \\
& v^{\prime}=v\left(v+\Delta v^{3}-u^{3}-2 B u^{2} v-B^{2} u v^{2}\right) .
\end{aligned}
$$

Taking $v=0$ the origin is the unique equilibrium point which is linearly zero. In order to understand the local phase portrait of this equilibrium point, we apply the directional blow-up $(u, v) \rightarrow(u, w)$ with $w=v / u$. And after eliminating the common factor $u$ between $u^{\prime}$ and $w^{\prime}$ we have

$$
\begin{aligned}
u^{\prime} & =-u\left(u^{2}+w+2 B u^{2} w+2 B w^{2}+B^{2} u^{2} w^{2}-\Delta u^{2} w^{3}\right), \\
w^{\prime} & =2 w^{2}(1+B w) .
\end{aligned}
$$

For $u=0$ system (25) has two possible equilibrium points $E_{1}=(0,0)$ and $E_{2}=$ $(0,-1 / B)$.

When $B=0$ the origin of system (25) is the unique equilibrium point on $u=0$ and it is linearly zero. Again we do another blow-up $(u, w) \rightarrow(u, \widetilde{w})$ with $\widetilde{w}=w / u$, remove the common factor $u$, and obtain the system

$$
u^{\prime}=-u\left(u+\widetilde{w}-\Delta u^{4} \widetilde{w}^{3}\right), \quad \widetilde{w}^{\prime}=\widetilde{w}\left(u+3 \widetilde{w}-\Delta u^{4} \widetilde{w}^{3}\right) .
$$




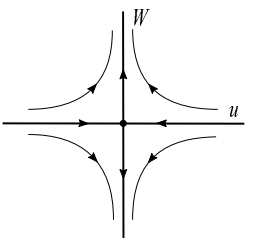

(a)

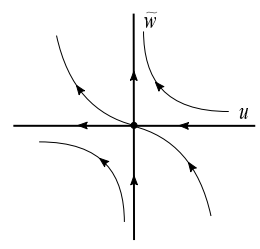

(b)

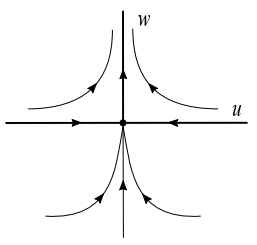

(c)

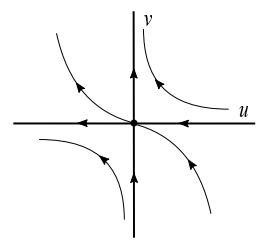

(d)

Figure 14. The blow-up at the origin of system (24) in $U_{1}$ with $B=0$. (a) System (27), (b) System (26), (c) System (25), (d) System (24).

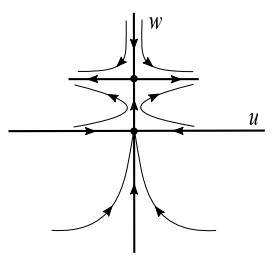

(a)

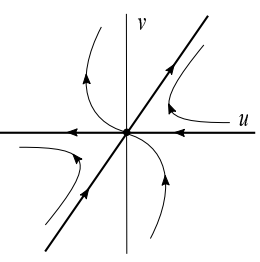

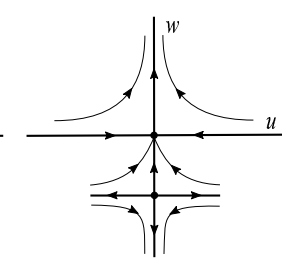

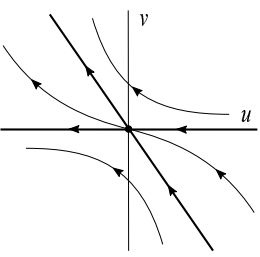

(b)

Figure 15. The blow-up at the origin of system (24) in $U_{1}$ with $B \neq 0$. (a) Systems (25) and (24) if $B<0$, (b) Systems (25) and (24) if $B>0$.

For $u=0$ the origin is the unique equilibrium, which is again linearly zero. We perform the directional blow-up $(u, \widetilde{w}) \rightarrow(u, W)$ with $W=\widetilde{w} / u$ and get the system

$$
\begin{aligned}
u^{\prime} & =-u\left(1+W-\Delta u^{6} W^{3}\right), \\
W^{\prime} & =W\left(1+u+3 W+u W-\Delta u^{6} W^{3}-\Delta u^{7} W^{3}\right) .
\end{aligned}
$$

after eliminating the common factor $u$. For $u=0$ system (27) has the origin as the unique equilibrium point, which is a hyperbolic saddle. Going back through the change of variables we get that locally $E_{1}$ has two hyperbolic sectors and one parabolic sector, see Figure 14(c). Again going back through the change of variables until system (24) and taking into account the behavior of the flow of system (24) on the axes, we have $\left.u^{\prime}\right|_{v=0}=-u^{4}$ and $\left.v^{\prime}\right|_{u=0}=v^{2}+O\left(v^{4}\right)$. Hence we obtain that the local phase portrait at the origin in $U_{1}$ has two hyperbolic sectors and two parabolic sectors see Figure 14(d).

Assume that $B \neq 0$. The equilibrium point $E_{1}$ is linearly zero and $E_{2}$ is a saddle. Similarly to the above case the local phase portrait of $E_{1}$ is shown in Figure 14(c). We superpose the analysis of the equilibria $E_{1,2}$, see the left of Figures 15 (a) and 15(b) when $B<0$ and $B>0$, respectively. Going back through the change of variables and taking into account the phase portrait of the flow on the axes, we obtain that the local phase portrait at the origin of $U_{1}$ also has two hyperbolic and two parabolic sectors (see the right of Figures 15(a) and 15(b)). 


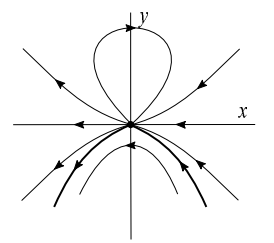

(a)

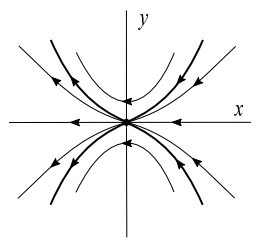

(b)

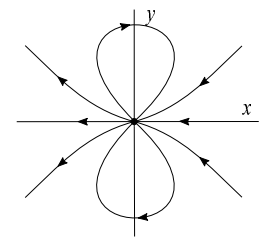

(c)

Figure 16. The local phase portrait at the origin of systems (23) and (30). (a) System (23) with $B=0$, (b) System (30) with $B>0$, (c) System (30) with $B<0$.

Now we check the origin of $U_{2}$, in which system (23) becomes

$$
\begin{aligned}
& u^{\prime}=1+2 B v+u^{2} v+B^{2} v^{2}+2 B u^{2} v^{2}-\Delta v^{3}, \\
& v^{\prime}=2 u v^{2}(1+B v) .
\end{aligned}
$$

The origin of $U_{2}$ is not the equilibrium point.

Thus the associated polynomial system (23) has two equilibria at infinity, one in $U_{1}$ and its diametrically opposite in $V_{1}$. Note that the degree of system (23) is 3 so the flow in the chart $V_{1}$ has the same sense as in $U_{1}$. The set $L=\{(x, y) \mid y+B=0\}$ in the Poincaré disk is a straight line connecting the origin in $U_{1}$ and $V_{1}$. We remark that in this case the origins in $U_{1}$ and $V_{1}$ are not equilibrium points for the rational Hamiltonian system (22) because they are in $L$.

This finishes the study of the infinite equilibrium points of system (23) and of the rational Hamiltonian system (22), we now focus on the finite region. Since the level of energy on the $x$-axis $\left.H_{21}^{+}\right|_{y=0}=x^{2}+\Delta / B=h$ has at most two solutions for any $h \in \mathbb{R}$, the integral curves of systems (22) and (23) cut the $x$-axis at most once at each region $x>0$ and $x<0$ when $B \neq 0$. And by the level of energy on the $y$-axis $\left.H_{21}^{+}\right|_{x=0}=y^{2} / 2+\Delta /(y+B)$, the integral curves of systems (22) and (23) cut the $y$-axis at most twice. Next we consider the following cases: (i) $\Delta=0$, (ii) $\Delta>0$ and (iii) $\Delta<0$.

(i.a) If $\Delta=0$ and $B=0$ system (23) has one finite equilibrium point $p_{1}=(0,0)$, which is linearly zero. Performing the directional blow-up $(x, y) \rightarrow(x, w)$ with $w=y / x$ and eliminating the common factor $x$, system (23) becomes

$$
x^{\prime}=x\left(-1+w^{3} x\right), \quad w^{\prime}=-w\left(1+w^{3} x\right) .
$$

When $x=0$ the origin is the unique equilibrium point of system (29), and it is an attracting node. Going back through the change of variables and taking into account the behavior of the flow on both axes, we get that locally the origin of systems (23) consists of one hyperbolic sector, one elliptic sector and two parabolic sectors (see Figure 16(a)). In fact the equilibrium point $p_{1}$ is a virtual equilibrium point of the rational Hamiltonian system (22). Thus there exist no finite equilibria for system (22). We obtain the phase portrait 1.2 of Figure 1.

(i.b) If $B \neq 0$ system (23) has two finite equilibrium points $p_{1}=(0,0)$ and $p_{2}=(0,-B)$. The eigenvalues of the equilibrium point $p_{1}$ are $\lambda_{1,2}= \pm \sqrt{-2 B^{3}}$. If $B>0$ the equilibrium $p_{1}$ is a center because system (23) is symmetric with 
the $y$-axis. The equilibrium point $p_{2}$ is linearly zero, doing the change of variables $x \rightarrow x$ and $y \rightarrow y-B$, we translate this equilibrium point to the origin and we have the system

$$
x^{\prime}=-x^{2}-B y^{2}+y^{3}, \quad y^{\prime}=-2 x y .
$$

Now we do the blow-up using $(x, y) \rightarrow(x, w)$ with $w=y / x$, and eliminate the common factor $x$ between $x^{\prime}$ and $w^{\prime}$. Then we have

$$
x^{\prime}=x\left(-1-B w^{2}+w^{3} x\right), \quad w^{\prime}=-w\left(1-B w^{2}+w^{3} x\right) .
$$

For $x=0$ the origin of system (31) is the unique equilibrium and it is an attracting node. Going back through the change of variables, taking into account the behavior of the flow on the axes of system (30), we have $\left.x^{\prime}\right|_{x=0}=-B y^{2}+O\left(y^{3}\right)$ and $\left.x^{\prime}\right|_{y=0}=-x^{2}$. Thus we get that the origin of system (30) has two hyperbolic and two parabolic sectors (see Figure 16(b)). Actually the equilibrium $p_{2}$ is a virtual equilibrium point of the associated system (22) because it is in the set $L$. Therefore there exists only one finite equilibrium for the rational Hamiltonian system (22). The phase portrait in this case is topologically equivalent to 1.3 of Figure 1.

If $B<0$ we have that $p_{1}$ is a saddle. Similarly to the above case we obtain that the origin of system (30) consists of two elliptic sectors and two parabolic sectors (see Figure 16(c)). On the other hand the equilibrium $p_{2}$ is a virtual equilibrium point of the Hamiltonian system (22), hence we have the phase portrait 1.4 of Figure 1.

Next we consider the case $\Delta \neq 0$, the explicit expressions for the finite equilibrium points of system (23) and their eigenvalues in terms of the parameters $b$ and $B$ are complicated. Hence we need firstly to find the number of finite equilibrium points for system (23). Using Theorems 2.2 and 2.4 we count the indices of known equilibrium point, then we can deduce the type of the remaining finite equilibrium points.

(ii) Assume that $\Delta>0$ system (23) has a equilibrium point of the form $(0, y)$ if $y$ satisfies

$$
y(y+B)^{2}-\Delta=0 .
$$

Let $\left(0, y_{0}\right)$ be an equilibrium point of system $(23)$, then the linear part of system (23) at $\left(0, y_{0}\right)$ is

$$
M=\left(\begin{array}{cc}
0 & \left(B+y_{0}\right)\left(B+3 y_{0}\right) \\
-2\left(B+y_{0}\right) & 0
\end{array}\right) .
$$

The equilibrium $\left(0, y_{0}\right)$ is linearly zero only if $B+y_{0}=0$ (i.e $\Delta=0$ ), which cannot be satisfied. We have that $\left(0, y_{0}\right)$ is either elementary or nilpotent, hence it is either a saddle, or a center, or a cusp. If $y_{0}=-B / 3$ we obtain that $\left(0, y_{0}\right)$ is nilpotent. Then we substitute $y_{0}=-B / 3$ into (32) and have $4 B^{3}+27 \Delta=0$.

The infinite equilibrium points in the Poincaré sphere are the origin of $U_{1}$, and also the corresponding point in $V_{1}$. The origins of $U_{1}$ and $V_{1}$ consist of two hyperbolic sectors respectively, by Theorem 2.2, they have index 0 . Thus the finite equilibrium points must have total index 2 in the Poincaré sphere. On the other hand, it is easy to get that the cubic equation (32) have one, or two, or three real roots if $4 B^{3} \Delta+27 \Delta>0$, or $4 B^{3} \Delta+27 \Delta=0$, or $4 B^{3} \Delta+27 \Delta<0$, respectively. Hence we have the following cases. 


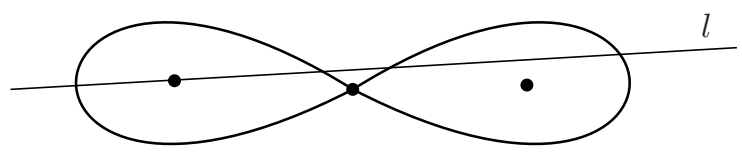

FiguRE 17. The straight line through the center intersects the separatrices four times.

(ii.a) If $4 B^{3}+27 \Delta>0$ system (23) has one finite equilibrium point, which is not in the set $L$. By Corollary 2.3 and Remark 2.5 this equilibrium point must be a center so the sum of the indices in the Poincaré sphere is 2 . Then we have the phase portrait 1.5 of Figure 1.

(ii.b) If $4 B^{3}+27 \Delta=0$ system (23) has two finite equilibrium points $p_{1}=$ $(0,-B / 3)$ and $p_{2}=(0,-4 B / 3)$, which are not in the set $L$. The equilibrium $p_{1}$ is a nilpotent point, and $p_{2}$ is a center because its eigenvalues are $\pm \sqrt{-9 \Delta / 2}$. Then according with Corollary 2.3 and Remark $2.5, p_{1}$ must be one cusp. In addition we have $0<-B / 3<-B<-4 B / 3$, then we obtain the phase portrait 1.6 of Figure 1 .

(ii.c) If $4 B^{3}+27 \Delta<0$ system (23) has three finite equilibrium points, there must be either just two centers and one saddle. Assume that the saddle is on the boundary of the period annulus of one center. We claim that this saddle cannot be on the boundary of the other center. If this were the case a straight line $l$ through the center passing sufficiently close to the saddle would have four intersection points with the separatrices which are on the same energy level as the saddle (two with the boundary of the period annulus of one center and two with the boundary of the other center), see Figure 17 for an illustration. This means that on the straight line $l$, which could be defined by $y=k x$ for some real number $k$, the equation $H_{21}^{+}=h$ would have four solutions. But this is not possible for the function $H_{21}^{+}$. Therefore the saddle on the boundary of the period annulus of one center has to be connected with the infinite equilibrium points. From $-2\left(B+y_{0}\right)^{2}\left(B+3 y_{0}\right)>0$ and $B<0$ we have $y_{0}<-B / 3$, and from $-2\left(B+y_{0}\right)^{2}\left(B+3 y_{0}\right)<0$ and $B<0$ we have $-B / 3<y_{0}<-B$ or $y_{0}>-B$. Then we get that the centers are in the intervals $(-B / 3,-B)$ or $(-B,+\infty)$, and the saddle is in the interval $(-\infty,-B / 3)$. In fact, by the continuity of the phase portraits, the cusp of the phase portrait 1.6 becomes the saddle of a center-loop. Hence the phase portrait of system (22) is topologically equivalent to the phase portrait 1.7 of Figure 1.

(iii) Assume that $\Delta<0$ then system (23) has two additional finite equilibrium points $p_{1,2}=( \pm \sqrt{-\Delta},-B)$ different from the equilibria $\left(0, y_{0}\right)$, which are an attracting node and a repelling node respectively. Hence each has index 1 . So the known equilibrium points have total index 4. By Theorem 2.4 the finite equilibrium points other than $p_{1,2}$ must have total index -1 in the Poincaré disk. On the other hand the equilibria $p_{1,2}$ are in the set $L$, they are two virtual points of the Hamiltonian system (22).

(iii.a) If $4 B^{3}+27 \Delta<0$ system (23) has one finite equilibrium point $p_{3}$ different than $p_{1,2}$, which is not in the straight line $y=-B$. According to Corollary 2.3 


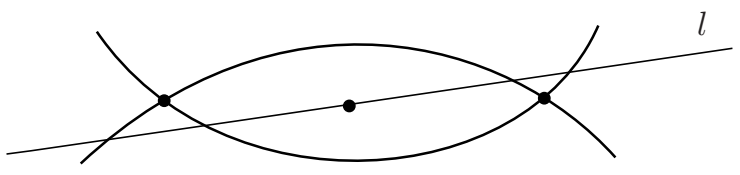

FiguRE 18. The straight line through the center intersects the separatrices four times.

the equilibrium point $p_{3}$ must be a saddle. Therefore the phase portrait of the Hamiltonian system (22) in this case is 1.8 of Figure 1.

(iii.b) If $4 B^{3}+27 \Delta=0$ system (23) has two finite equilibrium points $p_{3}=$ $(0,-B / 3)$ and $p_{4}=(0,-4 B / 3)$ which are not in set $L$. The equilibrium $p_{4}$ is a saddle because its eigenvalues are $\pm \sqrt{-9 \Delta / 2}$. The equilibrium point $p_{3}$ is a nilpotent point and it must be a cusp due to Corollary 2.3. In addition we have $0>-B / 3>-B>-4 B / 3$, then the phase portrait in this case is topologically equivalent to 1.9 of Figure 1.

(iii.c) If $4 B^{3}+27 \Delta>0$ system (23) has other three finite equilibrium points and they are not in the set $L$. By Corollary 2.3 and Remark 2.5, the finite equilibrium points must be two saddles and a center. Suppose first that all of the two saddles are on the boundary of the period annulus of the center. We could find a straight line $l$ through the center passing sufficiently close to the two saddles, which would have at least four intersection points with the separatrices which are on the same energy level, see Figure 18. Clearly this is not possible for the Hamiltonian $H_{21}^{+}$. From $-2\left(B+y_{0}\right)^{2}\left(B+3 y_{0}\right)>0$ and $B>0$ we have $y_{0}<-B$ or $-B<y_{0}<-B / 3$, and from $-2\left(B+y_{0}\right)^{2}\left(B+3 y_{0}\right)<0$ and $B>0$ we have $y_{0}>-B / 3$. Then we get that the center is in the interval $(-B / 3,+\infty)$, and the saddles are in the intervals $(-\infty,-B)$ or $(-B,-B / 3)$. Hence the phase portrait of the Hamiltonian system (22) in this case is topologically equivalent to 1.10 of Figure 1 . We conclude the proof of the theorem.

\section{Proof of Theorem 1.4}

We analyze the rational Hamiltonian system $H_{12}=\frac{y^{2}}{2}+\frac{x+b}{y^{2}+B y+C}$ given in statement (III) of Theorem 1.1, and the Hamiltonian system of $H_{12}$ is

$$
\dot{x}=y-\frac{(x+b)(2 y+B)}{\left(y^{2}+B y+C\right)^{2}}, \quad \dot{y}=-\frac{1}{y^{2}+B y+C} .
$$

Note that we can assume $B \geq 0$ because by the linear change $(x, y, t, b, B, C) \rightarrow$ $(x,-y,-t, b,-B, C)$ system $(34)$ is invariant. We will study the global phase portraits of system (34) when $C=B^{2} / 4$ and $C \neq B^{2} / 4$ separately.

(i) If $C=B^{2} / 4$ we apply the rescaling of the time $d t=(y+B / 2)^{3} d \tau$ and system (34) becomes

$$
x^{\prime}=-2(x+b)+y(y+B / 2)^{3}, \quad y^{\prime}=-(y+B / 2) .
$$




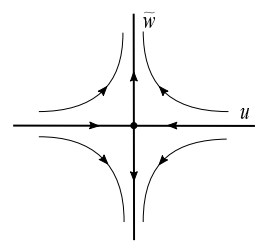

(a)

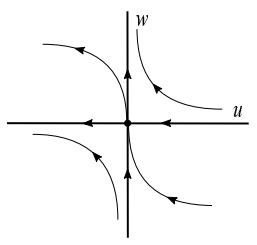

(b)

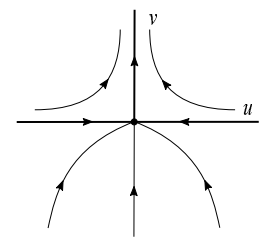

(c)

Figure 19. The blow-up at the origin of system (36) in $U_{1}$ with $B=0$. (a) System (38), (b) System (37), (c) System (36).

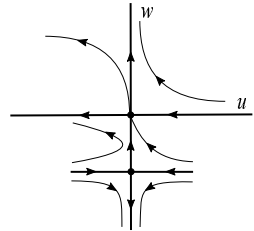

(a)

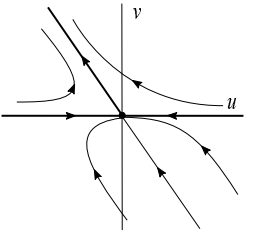

(b)

Figure 20. The blow-up at the origin of system (36) in $U_{1}$ with $B>0$. (a) System (37), (b) System (36).

System (35) in the local chart $U_{1}$ is

$$
\begin{aligned}
& u^{\prime}=-\frac{1}{8}\left(8 u^{5}+12 B u^{4} v+6 B^{2} u^{3} v^{2}-8 u v^{3}+B^{3} u^{2} v^{3}+4 B v^{4}-16 b u v^{4}\right), \\
& v^{\prime}=\frac{1}{8} v\left(16 v^{3}+16 b v^{4}-8 u^{4}-12 B u^{3} v-6 B^{2} u^{2} v^{2}-B^{3} u v^{3}\right) .
\end{aligned}
$$

For $v=0$ the origin is the unique equilibrium, which is linearly zero. In order to understand the local behavior of this equilibrium point we apply the directional blow-up $(u, v) \rightarrow(u, w)$ with $w=v / u$. And after eliminating the common factor $u^{3}$ we have

$$
\begin{aligned}
u^{\prime} & =-\frac{1}{8} u\left(8 u+12 B u w+6 B^{2} u w^{2}-8 w^{3}+B^{3} u w^{3}+4 B w^{4}-16 b u w^{4}\right), \\
w^{\prime} & =\frac{1}{2} w^{4}(2+B w) .
\end{aligned}
$$

Assume that $B=0$ system (37) has only one equilibrium point $E_{1}=(0,0)$ on $u=0$, and it is linearly zero. Again we do another blow-up $(u, w) \rightarrow(u, \widetilde{w})$ with $\widetilde{w}=w / u$, remove the common factor $u$, and obtain the system

$$
u^{\prime}=-\frac{u}{8}\left(8-8 u^{2} \widetilde{w}^{3}-16 b u^{4} \widetilde{w}^{4}\right), \quad \widetilde{w}^{\prime}=\frac{\widetilde{w}}{8}\left(8-16 b u^{4} \widetilde{w}^{4}\right)
$$

For $u=0$ the origin is the unique equilibrium, which is saddle. We recover the local behavior at the origin of system (36) going back through the blow-up, the equilibrium $E_{1}$ of system (37) has two hyperbolic sectors and two parabolic sectors (see Figure $19(\mathrm{~b})$ ), and the origin of $U_{1}$ consists of two hyperbolic and one attracting sectors (see Figure 19(c)). 


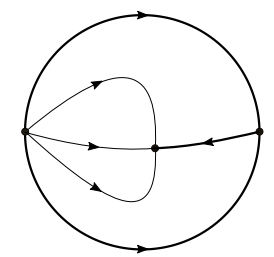

Figure 21. The phase portrait of system (35).

When $B>0$ system (37) has two equilibria $E_{1}$ and $E_{2}=(0,-2 / B)$ on $u=0$. The equilibrium $E_{1}$ is linearly zero and $E_{2}$ is a saddle. Similarly to the above case, we obtain that $E_{1}$ consists of two hyperbolic sectors and two parabolic sectors. We superpose the study of the equilibrium points $E_{1,2}$ of system (37), see Figure 20(a). Going back to the origin of $U_{1}$ and taking into account the behavior of the flow on the axes, we have $\left.u^{\prime}\right|_{u=0}=-B v^{4} / 2,\left.u^{\prime}\right|_{v=0}=-u^{5}$ and $\left.v^{\prime}\right|_{u=0}=2 v^{4}+O\left(v^{5}\right)$. Thus the origin of $U_{1}$ consists of two hyperbolic sectors and one parabolic sector (see Figure 20(b)).

In $U_{2}$ system (35) acquires the form

$$
\begin{aligned}
u^{\prime} & =\frac{1}{8}\left(8+12 B v+6 B^{2} v^{2}+B^{3} v^{3}-8 u v^{3}-16 b v^{4}+4 B u v^{4}\right), \\
v^{\prime} & =\frac{1}{2} v^{4}(2+B v),
\end{aligned}
$$

the origin is not the equilibrium point in $U_{2}$.

For the polynomial system (35) we have found two equilibria in the infinite region, one in $U_{1}$ and its diametrically opposite in $V_{1}$, which have the opposite sense. The set $L=\{(x, y) \mid y+B / 2=0\}$ is a straight line connecting the north and south poles. In this case the north and south poles are not equilibrium points for the rational Hamiltonian system (34) because they are in the set $L$.

To complete the global dynamics associated to $H_{21}$, we are going to analyze more properties of the level of energy. Using the energy relations $\left.H_{21}\right|_{y=0}=4(x+b) / B^{2}=$ $h$ and $\left.H_{21}\right|_{x=0}=y^{2} / 2+b /(y+B / 2)^{2}=h$, we have that the integral curves cross the $x$-axis at most once when $B \neq 0$ and cross the $y$-axis at most twice. Next we consider the finite equilibria for systems (35) and (34). System (35) has one finite equilibrium point $p_{1}=(-b,-B / 2)$, which is an attracting node. We get the phase portrait of system (35), see Figure 21. Going back to the Hamiltonian system (34), $p_{1}$ is a virtual equilibrium point and the integral curves of system (34) have the opposite orientation with respect to system (35) in the region $y<-B / 2$. Therefore in this case the phase portrait of the Hamiltonian system (34) is topologically equivalent to 1.11 of Figure 1.

(ii) Now we consider the case $C \neq B^{2} / 4$, and obtain the system

$$
x^{\prime}=-(x+b)(2 y+B)+y\left(y^{2}+B y+C\right)^{2}, \quad y^{\prime}=-\left(y^{2}+B y+C\right),
$$




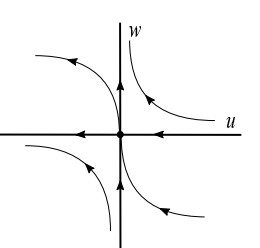

(a)

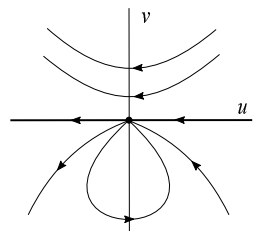

(b)

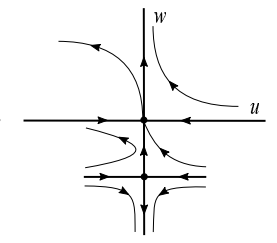

(c)

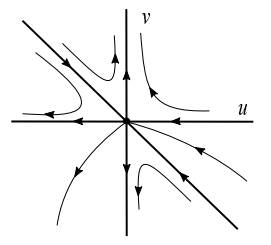

(d)

Figure 22. The blow-up at the origin of system (41) in $U_{1}$. (a) System (42) with $B^{2}<4 C$, (b) System (41) with $B^{2}<4 C$, (c) System (42) with $B>0$ and $C=0$, (d) System (41) with $B>0$ and $C=0$.

doing the rescaling $d t=\left(y^{2}+B y+C\right)^{2} d \tau$. In the chart $U_{1}$ system (40) becomes

$$
\begin{aligned}
u^{\prime}= & -u^{6}-2 B u^{5} v-\left(B^{2}+2 C\right) u^{4} v^{2}+u^{2} v^{3}-2 B C u^{3} v^{3}+\left(2 b-C^{2}\right) u^{2} v^{4} \\
& -C v^{5}+b B u v^{5}, \\
v^{\prime}= & v\left(-u^{5}-2 B u^{4} v-\left(B^{2}+2 C\right) u^{3} v^{2}+2 u v^{3}-2 B C u^{2} v^{3}+B v^{4}\right. \\
& \left.+\left(2 b-C^{2}\right) u v^{4}+b B v^{5}\right) .
\end{aligned}
$$

For $v=0$ the origin is the unique equilibrium point and it is linearly zero. We need to do the blow-up to understand the local phase portait in a neighborhood of the origin of system (41). We do the directional blow-up $(u, v) \rightarrow(u, w)$ with $w=v / u$ and have

$$
\begin{aligned}
u^{\prime}= & -u\left(u+2 B u w+B^{2} u w^{2}+2 C u w^{2}-w^{3}+2 B C u w^{3}\right. \\
& \left.-2 b u w^{4}+C^{2} u w^{4}+C w^{5}-b B u w^{5}\right), \\
w^{\prime}= & w^{4}\left(1+B w+C w^{2}\right),
\end{aligned}
$$

after eliminating the common factor $u^{4}$.

If $B^{2}-4 C<0$ the origin $E_{1}$ is the unique equilibrium point of system (42) on $u=0$, which is linearly zero. We perform the second blow-up $(u, w) \rightarrow(u, \widetilde{w})$ with $\widetilde{w}=w / u$ and eliminate the common factor $u^{4}$, then we have

$$
\begin{aligned}
u^{\prime}= & -u\left(1+2 B u \widetilde{w}+B^{2} u^{2} \widetilde{w}^{2}+2 C u^{2} \widetilde{w}^{2}-u^{2} \widetilde{w}^{3}+2 B C u^{3} \widetilde{w}^{3}\right. \\
& \left.-2 b u^{4} \widetilde{w}^{4}+C^{2} u^{4} \widetilde{w}^{4}+C u^{4} \widetilde{w}^{5}-b B u^{5} \widetilde{w}^{5}\right), \\
\widetilde{w}^{\prime}= & \widetilde{w}\left(1+2 B u \widetilde{w}+B^{2} u^{2} \widetilde{w}^{2}+2 C u^{2} \widetilde{w}^{2}+2 B C u^{3} \widetilde{w}^{3}\right. \\
& \left.+B u^{3} \widetilde{w}^{4}-2 b u^{4} \widetilde{w}^{4}+C^{2} u^{4} \widetilde{w}^{4}+2 C u^{4} \widetilde{w}^{5}-b B u^{5} \widetilde{w}^{5}\right) .
\end{aligned}
$$

For $u=0$ system (43) has the origin as the equilibrium point, and it is a saddle. Going back through the blow-up until system (41) in $U_{1}$ and taking into account the behavior of the flow on the axes, we have that $E_{1}$ has two hyperbolic sectors and two parabolic sectors (see Figure 22(a)), and the origin of system (41) consists of one elliptic, one hyperbolic and two parabolic sectors (see Figure 22(b)). In this case the separatrix must coincide with the $u$-axis, because it is invariant for system (41).

If $B>0$ and $C=0$ system (42) has two equilibrium points $E_{1}$ and $E_{2}=$ $(0,-1 / B)$ on $u=0$. Similarly to the above case $E_{1}$ is linearly zero which consists 


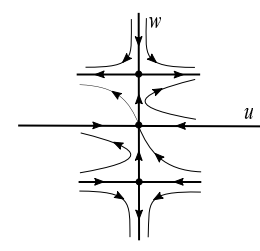

(a)

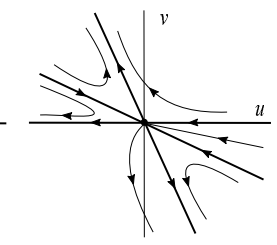

(b)

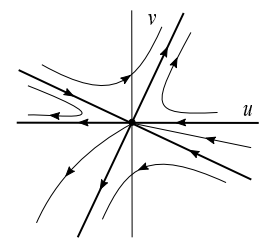

(c)

Figure 23. The blow-up at the origin of system (41) in $U_{1}$. (a) Systems (42) with $B^{2}>4 C$ and $C \neq 0$, (b) System (41) with $B^{2}>4 C>0$, (c) System (41) with $C<0$.

of two hyperbolic sectors and two parabolic sectors, and $E_{2}$ is a saddle (see Figure $22(\mathrm{c}))$. Going back through the blow-up and taking into account the behavior of the flow on the axes, we have $\left.u^{\prime}\right|_{v=0}=-u^{6}$ and $\left.v^{\prime}\right|_{u=0}=B v^{5}$. Then we see that the origin of $U_{1}$ in this case has four hyperbolic and two parabolic sectors (see Figure $22(\mathrm{~d}))$.

If $B^{2}>4 C$ and $C \neq 0$ system (42) has three equilibrium points $E_{1}$ and $E_{2,3}=$ $\left(0,\left(-B \pm \sqrt{B^{2}-4 C}\right) /(2 C)\right)$ on $u=0$. The equilibria $E_{2,3}$ are saddles. Similarly we obtain that the local behavior of $E_{1,2,3}$ as it is shown in Figure 23(a). Going back through the blow-up and taking into account the behavior of the flow on the axes, we have $\left.u^{\prime}\right|_{v=0}=-u^{6},\left.u^{\prime}\right|_{u=0}=-C v^{5}$ and $\left.v^{\prime}\right|_{u=0}=B v^{5}$. Therefore the origin of $U_{1}$ has also four hyperbolic and two parabolic sectors (see Figures 23(b) and $23(\mathrm{c}))$.

In $U_{2}$ system (40) acquires the form

$$
\begin{aligned}
& u^{\prime}=1+2 B v+\left(B^{2}+2 C\right) v^{2}+2 B C v^{3}-u v^{3}+\left(C^{2}-2 b\right) v^{4}-b B v^{5}+C u v^{5} \\
& v^{\prime}=v^{4}\left(1+B v+C v^{2}\right)
\end{aligned}
$$

the origin is not the equilibrium point in $U_{2}$.

Hence the associated polynomial system (40) has two equilibria in the infinite region, one in $U_{1}$ and its diametrically opposite in $V_{1}$, which have the same sense. When $B^{2}-4 C>0$ we observe that the set $L=\left\{(x, y) \mid y^{2}+B y+C=0\right\}$ corresponds to a couple of straight lines $y=\left(-B \pm \sqrt{B^{2}-4 C}\right) / 2$, which connect the north and south poles in the Poincaré disk. Therefore there exist no equilibrium at infinity for the rational system (34). If $B^{2}-4 C<0$ the set $L=\left\{(x, y) \mid y^{2}+B y+C=0\right\}$ is empty and the rational system (34) has two equilibria in the infinite region.

By the level of energy on the $x$-axis $\left.H_{12}\right|_{y=0}=h$ the integral curves, which have a point at the $x$-axis, cross the $x$-axis exactly once. And by the energy on the $y$-axis $\left.H_{12}\right|_{x=0}=y^{2} / 2+b /\left(y^{2}+B y+C\right)$, we have that the integral curves cross the $y$-axis at most twice. Now we study the finite equilibrium points of systems (34) and (40). If $B^{2}-4 C<0$ they have no equilibria. Therefore we obtain that the phase portraits of systems (34) and (40) are topologically equivalent to 1.12 of Figure 1. 
If $B^{2}-4 C>0$ system (40) has two equilibria points $p_{1,2}=(-b,(-B \pm$ $\left.\sqrt{B^{2}-4 C}\right) / 2$ ), which are an attracting node and a repelling node. The equilibrium points $p_{1,2}$ of system (40) are two virtual equilibrium points in the rational Hamiltonian system (34). Thus there exist no finite equilibria for system (34). The phase portrait of system (34) is topologically equivalent to 1.13 of Figure 1. Thus we conclude the proof of Theorem 1.4.

\section{Proof of Theorem 1.5}

Next we study the Hamiltonian system $H_{22}^{+}=\frac{y^{2}}{2}+\frac{x^{2}+\Delta}{y^{2}+B y+C}$, and the associated vector field of $H_{22}^{+}$is

$$
\dot{x}=y-\frac{\left(x^{2}+\Delta\right)(2 y+B)}{\left(y^{2}+B y+C\right)^{2}}, \quad \dot{y}=-\frac{2 x}{y^{2}+B y+C} .
$$

System (45) is invariant under the transformation

$$
(x, y, t, \Delta, B, C) \rightarrow(-x,-y, t, \Delta,-B, C) .
$$

Hence we only need to analyze the global phase portraits of system (45) with $B \geq 0$. It is easy to get that system (45) is symmetry with respect to the $y$-axis, in particular, it is symmetry with respect to the $x$-axis when $B=0$. We are going to study the dynamics for system (45) in two cases $C=B^{2} / 4$ and $C \neq B^{2} / 4$.

(i) In the case $C=B^{2} / 4$ we apply the rescaling $d t=(y+B)^{3} d \tau$ and have the system

$$
x^{\prime}=-2\left(x^{2}+\Delta\right)+y(y+B / 2)^{3}, \quad y^{\prime}=-2 x(y+B / 2) .
$$

Through the local chart we study the infinity of system (46) using the Poincaré compactification. The associated system (46) in the local chart $U_{1}$ is

$$
\begin{aligned}
& u^{\prime}=-\frac{1}{8}\left(8 u^{5}+12 B u^{4} v+6 B^{2} u^{3} v^{2}+8 B v^{3}+B^{3} u^{2} v^{3}-16 \Delta u v^{4}\right), \\
& v^{\prime}=\frac{1}{8} v\left(16 v^{2}+16 \Delta v^{4}-8 u^{4}-12 B u^{3} v-6 B^{2} u^{2} v^{2}-B^{3} u v^{3}\right) .
\end{aligned}
$$

For $v=0$ the origin is the unique equilibrium, which is linearly zero. In order to understand the local behavior of this equilibrium point, we apply the directional blow-up $(u, v) \rightarrow(u, w)$ with $w=v / u$. And after eliminating the common factor $u^{2}$ we have

$$
\begin{aligned}
& u^{\prime}=-\frac{1}{8}\left(8 u^{2}+12 B u^{2} w+6 B^{2} u^{2} w^{2}+8 B w^{3}+B^{3} u^{2} w^{3}-16 \Delta u^{2} w^{4}\right), \\
& w^{\prime}=\frac{1}{2} w^{3}(2+B w) .
\end{aligned}
$$

System (48) can have two possible equilibrium points $E_{1}=(0,0)$ and $E_{2}=$ $(0,-2 / B)$ on the $u=0$.

When $B=0$ the origin $E_{1}$ of system (48) is the unique equilibrium point for $u=0$ and it is a linearly zero. Again we do the blow-up $(u, w) \rightarrow(u, \widetilde{w})$ with $\widetilde{w}=w / u$, and after removing the common factor $u^{3}$ we obtain the system

$$
u^{\prime}=u\left(-1+2 \Delta u^{4} \widetilde{w}^{4}\right), \quad \widetilde{w}^{\prime}=\widetilde{w}\left(1+2 \widetilde{w}^{2}-2 \Delta u^{4} \widetilde{w}^{4}\right) .
$$

For $u=0$ system (49) has the origin as the unique equilibrium point, which is a saddle. We recover the local phase portrait at the origin of system (47) going back 


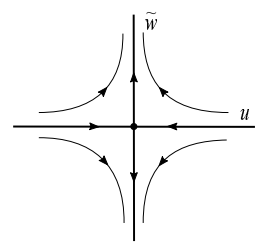

(a)

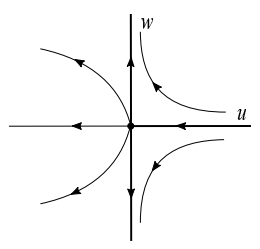

(b)

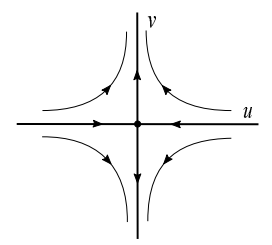

(c)

Figure 24. Blow-up at the origin of system (47) in $U_{1}$ with $B=0$.

(a) System (49), (b) System (48), (c) System (47).

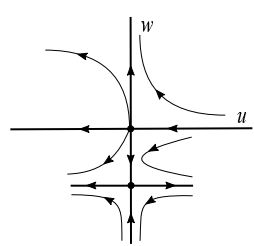

(a)

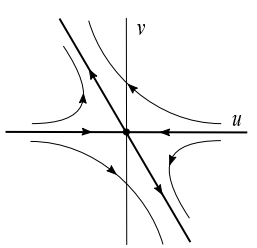

(b)

Figure 25. Blow-up at the origin of system (47) in $U_{1}$ with $B>0$.

(a) System (48), (b) System (47).

through the blow-up. The equilibrium $E_{1}$ of system (48) consists of two hyperbolic sectors and one repelling parabolic sector (see Figure 24(b)), the origin of $U_{1}$ has four hyperbolic sectors (see Figure 24(c)).

When $B>0$ system (48) has two equilibria $E_{1}$ and $E_{2}$ on the $u=0$. Similarly to the above case $E_{1}$ is a linearly zero equilibrium, which has two hyperbolic and one parabolic sectors. And the equilibrium $E_{2}$ is a saddle with eigenvalues $\lambda_{1,2}=$ $\pm 8 / B^{2}$. Going back through the blow-up and taking into account the behavior of the flow on the axes, we have $\left.u^{\prime}\right|_{u=0}=B v^{3},\left.u^{\prime}\right|_{v=0}=-u^{3}$ and $\left.v^{\prime}\right|_{u=0}=2 v^{3}+O\left(v^{4}\right)$. Then we obtain that the origin of system (47) also consists of four hyperbolic sectors see Figure 25.

We analyze the local chart $U_{2}$, system (46) in this chart is

(50) $u^{\prime}=\frac{1}{8}\left(8+12 B v+6 B^{2} v^{2}+B^{3} v^{3}+8 B u^{2} v^{3}-16 \Delta v^{4}\right), \quad v^{\prime}=u v^{3}(2+B v)$.

The origin is not the equilibrium point of system (50).

For the polynomial system (46) we have found two equilibria in the infinite region, one in $U_{1}$ and its diametrically opposite in $V_{1}$, which have the opposite sense. We observe that the set $L=\{(x, y) \mid y+B / 2=0\}$ corresponds to $y=-B / 2$, which is a straight line connecting the north and south poles. Therefore there exist no equilibria at infinity for the rational Hamiltonian system (45).

Now we study the finite equilibria of systems (45) and (46), and consider the following subcases (i.a) $\Delta=0$, (i.b) $\Delta>0$ and (i.c) $\Delta<0$. 


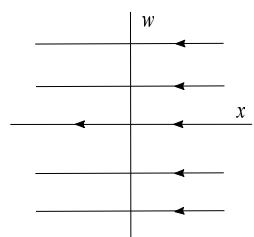

(a)

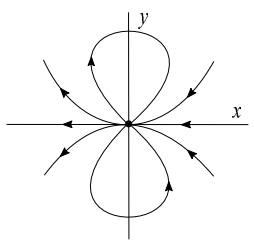

(b)

FiguRE 26. Blow-up at the origin of system (46) with $\Delta=B=0$.

(a) System (51), (b) System (46).

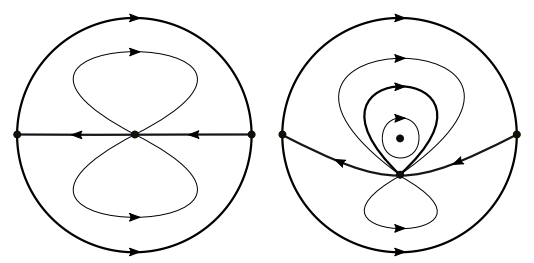

(a)

(b)

Figure 27. The phase portraits of system (46) with $\Delta=0$, (a) if $B=0$, (b) if $B>0$.

(i.a) If $\Delta=B=0$ system (46) has only one finite equilibrium point $p_{1}=(0,0)$, which is in the set $L$. And it is linearly zero. We preform the directional blow-up $(x, y) \rightarrow(x, w)$ with $w=y / x$ and eliminating the common factor $x^{2}$ we have

$$
x^{\prime}=-2+x^{2} w^{4}, \quad w^{\prime}=-x w^{5} .
$$

For $x=0$ this system does not have any equilibrium points, therefore we analyze the vector field in a neighborhood of the origin. Note that $\left.x^{\prime}\right|_{w=0}=-2$ and $\left.w^{\prime}\right|_{x=0}=0$, then the local phase portrait at the origin of system (51) is given in Figure 26(a). We reconstruct the flow through the blow-up to get the behavior of the flow close to the origin of system (46), it has two elliptic sectors and two parabolic sectors, one attracting and one repelling, Figure 26(b) shows this local phase portrait.

Hence we obtain the phase portrait of system (46), see Figure 27(a). Going back to the Hamiltonian system (45), $p_{1}$ is a virtual equilibrium point. By the rescaling time $d t=x^{3} d \tau$ the integral curves of system (45) has the opposite sense with respect to the ones of system (46) in the region $y<0$. Thus we have the phase portrait 1.14 of Figure 1.

If $\Delta=0$ and $B>0$ system (46) has two finite equilibria $p_{1}=(0,0)$ and $p_{2}=(0,-B / 2)$, where $p_{2}$ is in the set $L$ and it is linearly zero. The eigenvalues of $p_{1}$ are $\pm B^{2} \sqrt{2} i / 2$ and system (46) is symmetry with respect to the $y$-axis, hence $p_{1}$ is a center. We translate the equilibrium point $p_{2}$ to the origin though the change of variables $(x, y) \rightarrow(u, v-B / 2)$, and we have the system

$$
u^{\prime}=-\frac{1}{2}\left(4 u^{2}+B v^{3}-2 v^{4}\right), \quad v^{\prime}=-2 u v .
$$




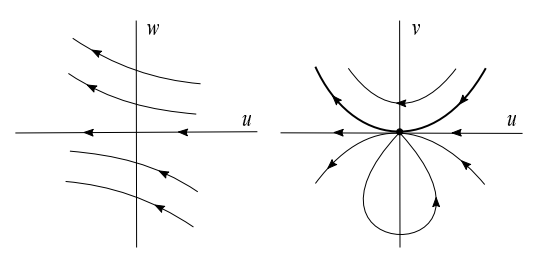

(a)

(b)

Figure 28. Blow-up at the origin of system (52). (a) System (53), (b) System (52).

Applying the directional blow-up $(u, v) \rightarrow(u, w)$ with $w=v / u$ and eliminating the common factor $u^{2}$ we have

$$
u^{\prime}=-\frac{1}{2}\left(4+B w^{3} u-2 w^{4} u^{2}\right), \quad w^{\prime}=\frac{1}{2} w^{4}(B-2 w u) .
$$

System (53) does not have any equilibrium points on $u=0$, hence we analyze the vector fields in a neighborhood of the origin. Note that $\left.u^{\prime}\right|_{w=0}=-2$ and $\left.w^{\prime}\right|_{u=0}=B w^{4} / 2>0$, then the local phase portrait at the origin of system (53) is given in Figure 28(a). We reconstruct the flow through the blow-up to get the local phase portrait of the flow at the origin of system (52). It has one elliptic, one hyperbolic and two parabolic sectors, see Figure 28(b).

In this subcase we have the phase portrait of system (46), as it is shown in Figure 27(b). Going back to the Hamiltonian system (45), $p_{2}$ is a virtual equilibrium point and the integral curves of system (45) have the opposite sense with respect to the ones of system (46) in the region $y<-B / 2$. Hence the phase portrait is topologically equivalent to 1.15 of Figure 1.

(i.b) If $\Delta>0$ system (46) has an equilibrium point of the form $(0, y)$ if $y$ satisfies

$$
8 y^{4}+12 B y^{3}+6 B^{2} y^{2}+B^{3} y-16 \Delta=0 .
$$

Let $\left(0, y_{0}\right)$ be an equilibrium point of system $(46)$, which is not in the set $L$. Then the linear part of $(46)$ at $\left(0, y_{0}\right)$ is

$$
M=\left(\begin{array}{cc}
0 & \frac{1}{8}\left(B+2 y_{0}\right)^{2}\left(B+8 y_{0}\right) \\
-\left(B+2 y_{0}\right) & 0
\end{array}\right) .
$$

The equilibrium point $\left(0, y_{0}\right)$ is linearly zero only if $y_{0}+B / 2=0$, then we have $\Delta=0$ from (46), which cannot be satisfied. It is a nilpotent point only if $y_{0}=$ $-B / 8$, we substitute this condition into (54) and obtain $8192 \Delta+27 B^{4}=0$, which also cannot be satisfied. Hence we have that $\left(0, y_{0}\right)$ is an elementary equilibrium, and it is either a saddle or a center.

Now we analyze the number roots of equation (54) and compute the discriminant sequence $\left[D_{1}, D_{2}, D_{3}, D_{4}\right]$ where

$$
\begin{aligned}
& D_{1}=256, \quad D_{2}=3072 B^{2}, \quad D_{3}=1572864 \Delta B^{2}, \\
& D_{4}=-4194304 \Delta^{2}\left(8192 \Delta+27 B^{4}\right) .
\end{aligned}
$$

Observe that we have $D_{1}>0$, and $D_{4}<0$ because $\Delta>0$, hence the sign list of this discriminant sequence is determined only by the signs of $B$. It is given in Theorem 


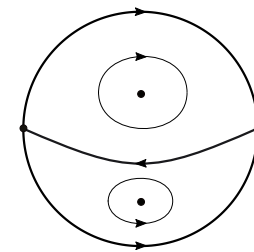

(a)

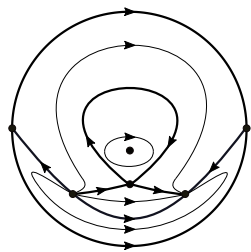

(b)

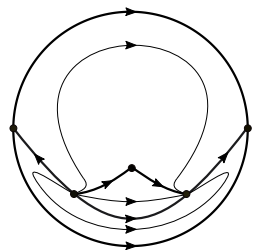

(c)

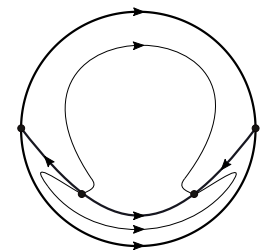

(d)

Figure 29. The phase portraits of system (46), (a) if $\Delta>0$, (b) if $B>0$ and $-27 B^{4} / 8192<\Delta<0$, (c) if $B>0$ and $\Delta=-27 B^{4} / 8192$, (d) if $\Delta<-27 B^{4} / 8192$.

2.1 that the possible revised sign lists that we need to study are $[1,1,1,-1]$ and $[1,-1,-1,-1]$, which correspond to $B>0$ and $B=0$ respectively. From Theorem 2.1 we have that equation (54) has two real distinct roots, accordingly system (46) has two finite equilibrium points, which are not in the $L$.

We continue determining the finite equilibria of systems (46) and (45). The infinite equilibrium points in the Poincaré sphere are the origin in $U_{1}$, and also the corresponding point in $V_{1}$. The origins of $U_{1}$ and $V_{1}$ consist of four hyperbolic sectors, hence they have index -2 by Theorem 2.2. Thus, in the Poincaré disk, the finite equilibrium points must have total index 2 . Note that the two remaining finite equilibrium points are $p_{1,2}$, in particularly $p_{1,2}=(0, \pm \sqrt[4]{2 \Delta})$ when $B=0$. By Corollary 2.3 and Remark 2.5 the equilibria $p_{1,2}$ must be centers. In this subcase we have the phase portrait of system (46), see Figure 29(a). Going back to the Hamiltonian system (45), the integral curves of system (45) have the opposite sense with respect to the ones of system (46) in the region $y<-B / 2$. Hence in this subcase we have the phase portrait 1.16 of Figure 1.

(i.c) Assume that $\Delta<0$ system (46) has two additional finite equilibria $p_{1,2}=$ $( \pm \sqrt{-\Delta},-B / 2)$ in the set $L$, which are distinct to the points from (54). The equilibrium points $p_{1,2}$ are an attracting node and a repelling node, hence each has index 1. So the known equilibrium points have total index 2. By Theorem 2.4 the remaining finite equilibrium points must have total index 0 . We will determine the cases in which (54) has two, one or zero distinct real roots. These roots correspond the equilibria of systems (45) and (46), which are not in the set $L$.

According with Theorem 2.1 equation (54) has two distinct real roots if and only if the revised sign list of its discriminant sequence is $[1,1,-1,-1]$ because we have $D_{3} \leq 0$. Hence we have $B>0$ and $-27 B^{4} / 8192<\Delta<0$. Then system (46) has two additional finite equilibrium points $p_{3,4}$ if and only if $B>0$ and $-27 B^{4} / 8192<\Delta<0$. From Corollary 2.3 the equilibria $p_{3,4}$ must be a saddle and a center so that they have total index 0 . By $-\left(B+y_{0}\right)^{3}\left(B+8 y_{0}\right) / 8>0$ and $B>0$ we have $-B / 2<y_{0}<-B / 8$, and from $-\left(B+y_{0}\right)^{3}\left(B+8 y_{0}\right) / 8<0$ and $B>0$ we have $y_{0}>-B / 8$ or $y_{0}<-B / 2$. Then we get that the center is in the interval $(-B / 8,+\infty)$ or $(-\infty,-B / 2)$, and the saddle is in the interval $(-B / 2,-B / 8)$. In this subcase we have the phase portrait of system (46), see Figure 29(b). Going back to the Hamiltonian system (45), $p_{1,2}$ are two virtual equilibrium points and the integral curves of system (45) have the opposite sense with respect to the ones 


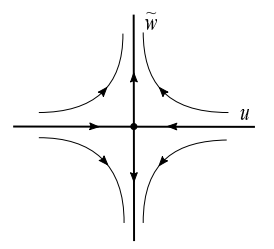

(a)

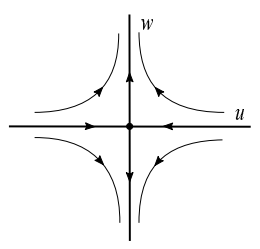

(b)

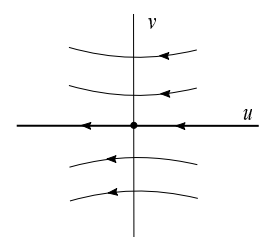

(c)

Figure 30. Blow-up at the origin of system (57) in $U_{1}$ with $C>$ $B^{2} / 4$. (a) System (59), (b) System (58), (c) System (57).

of system (46) in the region $y<-B / 2$. Hence the phase portrait of system (45) is topologically equivalent to 1.17 of Figure 1.

Similarly equation (54) has one distinct real root if and only if the revised sign list is $[1,1,-1,0]$, i.e $B>0$ and $\Delta=-27 B^{4} / 8192$. This root corresponds to an equilibrium of systems (45) and (46). By Corollary 2.3 this equilibrium must be a cusp. Hence we have the phase portrait of system (46), see Figure 29(c). Going back to the Hamiltonian system (45) we have that the phase portrait is topologically equivalent to 1.18 of Figure 1.

When $\Delta<-27 B^{4} / 8192$ we have that the revised sign list is $[1,1,-1,1]$ or $[1,-1,-1,1]$. Thus system (46) has no finite equilibrium points which are not in the set $L$. Hence we have the phase portrait of system (46), see Figure 29(d). Similarly to the above case we go back to the Hamiltonian system (45), and have the phase portrait 1.19 of Figure 1.

(ii) Next we consider the case $C \neq B^{2} / 4$, then system (45) becomes

(56) $x^{\prime}=-\left(x^{2}+\Delta\right)(2 y+B)+y\left(y^{2}+B y+C\right)^{2}, \quad y^{\prime}=-2 x\left(y^{2}+B y+C\right)$,

after the rescaling time $d t=\left(y^{2}+B y+C\right)^{2} d \tau$. And system (56) in the chart $U_{1}$ is

$$
\begin{aligned}
u^{\prime}= & -u^{6}-2 B u^{5} v-B^{2} u^{4} v^{2}-2 C u^{4} v^{2}-B u v^{3}-2 B C u^{3} v^{3}-2 C v^{4} \\
& +2 \Delta u^{2} v^{4}-C^{2} u^{2} v^{4}+\Delta B u v^{5} \\
v^{\prime}= & v\left(-u^{5}-2 B u^{4} v+2 u v^{2}-B^{2} u^{3} v^{2}-2 C u^{3} v^{2}+B v^{3}-2 B C u^{2} v^{3}\right. \\
& \left.+2 \Delta u v^{4}-C^{2} u v^{4}+\Delta B v^{5}\right)
\end{aligned}
$$

For $v=0$ the origin is the unique equilibrium and it is linearly zero. Doing the directional blow-up $(u, v) \rightarrow(u, w)$ with $w=v / u$ and eliminating the common factor $u^{3}$, system (57) becomes

$$
\begin{aligned}
u^{\prime}= & -u\left(u^{2}+2 B u^{2} w+B^{2} u^{2} w^{2}+2 C u^{2} w^{2}+B w^{3}+2 B C u^{2} w^{3}+2 C w^{4}\right. \\
& \left.-2 \Delta u^{2} w^{4}+C^{2} u^{2} w^{4}-\Delta B u^{2} w^{5}\right) \\
v^{\prime}= & 2 w^{3}\left(1+B w+C w^{2}\right) .
\end{aligned}
$$

When $C>B^{2} / 4$ the equilibrium point $E_{1}=(0,0)$ of system $(58)$ is the unique equilibrium point on $u=0$ and it is linearly zero. We do another blow-up $(u, w) \rightarrow$ 


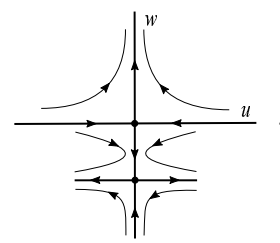

(a)

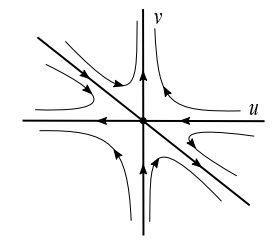

(b)

Figure 31. Blow-up at the origin of system (57) in $U_{1}$ with $C=0$ and $B>0$. (a) System (58), (b) System (57).

$(u, \widetilde{w})$ with $\widetilde{w}=w / u$, removing the common factor $u^{2}$, we have

$$
\begin{aligned}
u^{\prime}= & u\left(-1-2 B u \widetilde{w}-B^{2} u^{2} \widetilde{w}^{2}-2 C u^{2} \widetilde{w}^{2}-B u \widetilde{w}^{3}-2 B C u^{3} \widetilde{w}^{3}-2 C u^{2} \widetilde{w}^{4}\right. \\
& \left.+2 \Delta u^{4} \widetilde{w}^{4}-C^{2} u^{4} \widetilde{w}^{4}+\Delta B u^{5} \widetilde{w}^{5}\right), \\
\widetilde{w}^{\prime}= & \widetilde{w}\left(1+2 B u \widetilde{w}+2 \widetilde{w}^{2}+B^{2} u^{2} \widetilde{w}^{2}+2 C u^{2} \widetilde{w}^{2}+3 B u \widetilde{w}^{3}+2 B C u^{3} \widetilde{w}^{3}\right. \\
& \left.+4 C u^{2} \widetilde{w}^{4}-2 \Delta u^{4} \widetilde{w}^{4}+C^{2} u^{4} \widetilde{w}^{4}-\Delta B u^{5} \widetilde{w}^{5}\right) .
\end{aligned}
$$

For $u=0$ the origin is the unique equilibrium point of system (59), which is a saddle. We recover the local phase portrait at the origin of system (58) going back through the blow-up, the equilibrium $E_{1}$ of system (58) consists of four hyperbolic sectors, as it is shown in Figure 30(b). Again going back through the blow-up until system (57) and taking into account the flow on the axes, we obtain that the origin of $U_{1}$ has two hyperbolic sectors see Figure 30(c), and the $u$-axis contains two separatrices because it is invariant for system (57).

If $C=0$ and $B>0$ system (58) has two equilibria $E_{1}$ and $E_{2}=(0,-1 / B)$ on $u=0$. From system (58) it is easy to get that $E_{2}$ is a saddle. Similarly to the above case we obtain that in a neighborhood of the equilibrium $E_{1}$ has four hyperbolic sectors doing a blow-up. In Figure 31(a) we superpose the study of the two equilibria $E_{1,2}$ obtained by the blow-up at the origin of system (57). Going back through this blow-up and taking into the behavior of the flow on both axes, we obtain that the local phase portrait at the origin of system (58) consists of six hyperbolic sectors, see Figure 31(b).

Similarly, if $C<B^{2} / 4$ and $C \neq 0$ system (58) has three equilibria $E_{1}$ and $E_{3,4}=\left(0,\left(-B \pm \sqrt{B^{2}-4 C}\right) /(2 C)\right)$ on $u=0$. Proceeding analogously as in the above study we obtain that the local phase portrait at the origin of systems (58) consists of six hyperbolic sectors see Figures $32(\mathrm{~b})$ and $32(\mathrm{c})$ when $C>0$ and $C<0$.

In the local chart $U_{2}$ system (56) becomes

$$
\begin{aligned}
u^{\prime}= & +2 B v+B^{2} v^{2}+2 C v^{2}+2 B C v^{3}+B u^{2} v^{3}-2 \Delta v^{4}+C^{2} v^{4} \\
& +2 C u^{2} v^{4}-\Delta B v^{5} \\
v^{\prime}= & 2 u v^{3}\left(1+B v+C v^{2}\right),
\end{aligned}
$$

the origin is not the equilibrium point of system (60). 


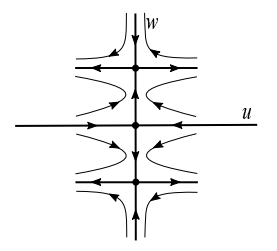

(a)

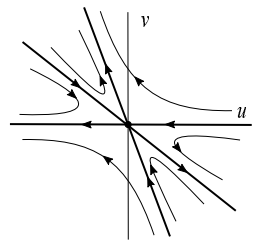

(b)

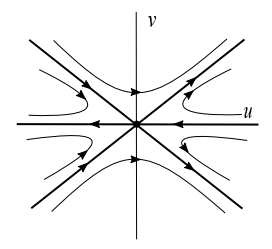

(c)

Figure 32. Blow-up at the origin of system (57) in $U_{1}$ with $C<$ $B^{2} / 4$. (a) System (58), (b) System (57) with $0<C$, (c) System (57) with $C<0$.

For the polynomial system (56) we have found two equilibria at infinity, one in $U_{1}$ and its diametrically opposite in $V_{1}$, which have the same sense. When $B^{2}-4 C>0$ we observe that the set $L=\left\{(x, y) \mid y^{2}+B y+C=0\right\}$ corresponds to two straight lines $y_{1,2}=\left(-B \pm \sqrt{B^{2}-4 C}\right) / 2$, which connect the north and south poles. Therefore there exist no equilibria at infinity for the rational Hamiltonian system (45). If $B^{2}-4 C<0$ the set $L=\left\{(x, y) \mid y^{2}+B y+C=0\right\}$ is empty, system (45) has two equilibria in the infinite region.

This finishes the study of the infinite equilibrium points of system (56) and of the rational Hamiltonian system (45), we now focus on the finite region in three subcases: $\Delta>0, \Delta=0$ and $\Delta<0$.

(ii.a) If $\Delta>0$ system (56) has an equilibrium point of the form $(0, y)$ if $y$ satisfies $g(y)=0$ where

$$
g(y)=y^{5}+2 B y^{4}+\left(2 C+B^{2}\right) y^{3}+2 B C y^{2}+\left(C^{2}-2 \Delta\right) y-\Delta B
$$

Let $\left(0, y_{0}\right)$ be an equilibrium point of system (56), then the linear part of (56) at $\left(0, y_{0}\right)$ is

$$
\left(\begin{array}{cc}
0 & F_{2} \\
-2 F_{1} & 0
\end{array}\right)
$$

where $F_{1}=y_{0}^{2}+B y_{0}+C$ and

$$
F_{2}=5 y_{0}^{4}+8 B y_{0}^{3}+\left(3 B^{2}+6 C\right) y_{0}^{2}+4 B C y_{0}-2 \Delta+C^{2}
$$

Since $\Delta>0$ and $C \neq B^{2} / 4$ we have $F_{1} \neq 0$ from (56). Hence the equilibrium $\left(0, y_{0}\right)$ is either elementary or nilpotent, that is, it is either a saddle or a center or a cusp, and it is not in the set $L$.

Now we will analyze the number of real roots of (61). The roots correspond the equilibrium points for systems (56) and (45). The elements of the discriminant 
sequence of (61) are

$$
\begin{aligned}
D_{1}= & 5, \quad D_{2}=2\left(3 B^{2}-10 C\right), \\
D_{3}= & 4\left(12 \Delta B^{2}-40 \Delta C+B^{2} C^{2}-4 C^{3}\right), \\
D_{4}= & -2 \Delta\left(1280 \Delta^{2}-45 \Delta B^{4}+326 \Delta B^{2} C-512 \Delta C^{2}\right. \\
& \left.-4 B^{4} C^{2}+32 B^{2} C^{3}-64 C^{4}\right), \\
D_{5}= & D_{5}(\Delta, B, C)=-\Delta^{2}\left(8192 \Delta^{3}-837 \Delta^{2} B^{4}+108 \Delta B^{8}+6528 \Delta^{2} B^{2} C\right. \\
& -1404 \Delta B^{6} C-12288 \Delta^{2} C^{2}+6432 \Delta B^{4} C^{2}-11712 \Delta B^{2} C^{3}+16 B^{6} C^{3} \\
& \left.+6144 \Delta C^{4}-192 B^{4} C^{4}+768 B^{2} C^{5}-1024 C^{6}\right) .
\end{aligned}
$$

(ii.a.1) On the basis of Theorem 2.1 we obtain that the polynomial (54) has five distinct real roots if and only if the revised sign list of its discriminant sequence is $[1,1,1,1,1]$. Hence we have $D_{i}>0$ for $i=1,2, \cdots, 5$. In this subcase we have assumed $B \geq 0, \Delta>0$ and $C \neq B^{2} / 4$. From $D_{2}>0$ and $D_{3}>0$ we get that it needs to satisfy either $C<B^{2} / 4$, or $B^{2} / 4<C<3 B^{2} / 10$ and $\Delta>$ $C^{2}\left(4 C-B^{2}\right) /\left(4\left(3 B^{2}-10 C\right)\right)>0$. Letting $F_{3}=C^{2}\left(4 C-B^{2}\right) /\left(4\left(3 B^{2}-10 C\right)\right)$. And from $D_{4}>0$ we get $0<\Delta<F_{4}$ where

$$
\begin{aligned}
& F_{4}=\frac{45 B^{4}-326 B^{2} C+512 C^{2}}{2560}+\frac{3 \sqrt{F_{5}}}{2560}, \\
& F_{5}=225 B^{8}-3260 B^{6} C+19204 B^{4} C^{2}-55296 B^{2} C^{3}+65536 C^{4} .
\end{aligned}
$$

Actually $F_{4}>0$ and $F_{5}>0$, because we have

$$
\left(45 B^{4}-326 B^{2} C+512 C^{2}\right)^{2}-9 F_{5}=-20480\left(B^{2}-4 C\right)^{2} C^{2} \leq 0
$$

and $\left.F_{4}\right|_{C=0}=9 B^{4} / 256>0$. On the other hand, we have $F_{3}>F_{4}$ in the case $B^{2} / 4<C<3 B^{2} / 10$, hence it just needs to prove

$$
\begin{aligned}
& 2560 C^{2}\left(4 C-B^{2}\right)-4\left(3 B^{2}-10 C\right)\left(45 B^{4}-326 B^{2} C+512 C^{2}+3 \sqrt{F_{5}}\right) \\
= & -12\left(45 B^{6}-476 B^{4} C+1812 B^{2} C^{2}-2560 C^{3}+\left(3 B^{2}-10 C\right) \sqrt{F_{5}}\right)>0 .
\end{aligned}
$$

In fact we have

$$
\begin{aligned}
& \left(45 B^{6}-476 B^{4} C+1812 B^{2} C^{2}-2560 C^{3}\right)^{2}-\left(3 B^{2}-10 C\right)^{2} F_{5} \\
= & -1280 B^{2}\left(B^{2}-6 C\right)^{2}\left(B^{2}-4 C\right) C^{2}>0
\end{aligned}
$$

and

$$
45 B^{6}-476 B^{4} C+1812 B^{2} C^{2}-2560 C^{3}<0 .
$$

Then we have $C<B^{2} / 4$ and $0<\Delta<F_{4}$ from $D_{1,2,3,4}>0$. Furthermore (61) has five real roots if and only if $B=0, C<0$ and $0<\Delta<C^{2} / 2$, or $B>0, C<B^{2} / 4$, $0<\Delta<F_{4}$ and $D_{5}>0$. Accordingly system (56) has five finite equilibrium points, which are not in the set $L$. 
Now we analyze the location of the five distinct real roots. Let $g(x)$ be a polynomial of degree $d(g(x))=n$, and we define the following polynomials

$$
\begin{aligned}
g_{0}(x) & :=g(x), \\
g_{1}(x) & :=g^{\prime}(x), \\
g_{0}(x) & =: g_{1}(x) q_{1}(x)-\lambda_{2} g_{2}(x), \\
g_{1}(x) & =: g_{2}(x) q_{2}(x)-\lambda_{3} g_{3}(x), \\
& \cdots \\
g_{m-2}(x) & =: g_{m-1}(x) q_{m-1}(x)-\lambda_{m} g_{m}(x), \\
g_{m-1}(x) & =: g_{m}(x) q_{m}(x),
\end{aligned}
$$

where $\lambda_{i}>0, d\left(g_{i}(x)\right)<d\left(g_{i-1}(x)\right)$ and $d\left(g_{m}(x)\right)=0$.

Theorem 7.1 (Sturm Theorem). Assume that $g_{0}(x), g_{1}(x), \cdots, g_{m}(x)$ are as the polynomials (65) where $g(a) g(b) \neq 0$. For each $x \in \mathbb{R}, V(x)$ denotes the number of the sign changes of the sequence $\left\{g_{0}(x), g_{1}(x), \cdots, g_{m}(x)\right\}$. We have that the number of real roots of $g(x)$ in $(a, b)$ is $V(a)-V(b)$.

Using the above Sturm criterion we take the polynomial $g_{0}(y)=g(y)$ from $(61)$, and from (65) we obtain

$$
\begin{aligned}
g_{1}(y)= & 5 y^{4}+8 B y^{3}+3\left(B^{2}+2 C\right) y^{2}+4 B C y-2 \Delta+C^{2}, \\
g_{2}(y)= & \left(6 B^{2}-20 C\right) y^{3}+\left(6 B^{3}-18 B C\right) y^{2}+\left(40 \Delta+8 B^{2} C-20 C^{2}\right) y \\
& +21 \Delta B+2 B C^{2}, \\
g_{3}(y)= & \left(24 \Delta B^{2}-80 \Delta C+2 B^{2} C^{2}-8 C^{3}\right) y^{2}+\left(27 \Delta B^{3}-98 \Delta B C+2 B^{3} C^{2}\right. \\
& \left.-8 B C^{3}\right) y+9 \Delta B^{4}-39 \Delta B^{2} C+16 \Delta C^{2}+2 B^{2} C^{3}-8 C^{4}, \\
g_{4}(y)= & \left(-1280 \Delta^{2}+45 \Delta B^{4}-326 \Delta B^{2} C+512 \Delta C^{2}+4 B^{4} C^{2}-32 B^{2} C^{3}\right. \\
& \left.+64 C^{4}\right) y-672 \Delta^{2} B-9 \Delta B^{5}+87 \Delta B^{3} C-240 \Delta B C^{2}, \\
g_{5}(y)= & 8192 \Delta^{3}-837 \Delta^{2} B^{4}+108 \Delta B^{8}+6528 \Delta^{2} B^{2} C-1404 \Delta B^{6} C \\
& -12288 \Delta^{2} C^{2}+6432 \Delta B^{4} C^{2}-11712 \Delta B^{2} C^{3}+16 B^{6} C^{3}+6144 \Delta C^{4} \\
& -192 B^{4} C^{4}+768 B^{2} C^{5}-1024 C^{6} .
\end{aligned}
$$

We have that under conditions $C<B^{2} / 4,0<\Delta<F_{4}$ and $D_{5}>0$ the polynomial

TABLE 1

\begin{tabular}{ccccc}
\hline$y$ & $-\infty$ & $\frac{-B-\sqrt{B^{2}-4 C}}{2}$ & $\frac{-B+\sqrt{B^{2}-4 C}}{2}$ & $\infty$ \\
\hline $\operatorname{sign}\left(g_{0}(y)\right)$ & - & + & - & + \\
$\operatorname{sign}\left(g_{1}(y)\right)$ & + & - & - & + \\
$\operatorname{sign}\left(g_{2}(y)\right)$ & - & - & + & + \\
$\operatorname{sign}\left(g_{3}(y)\right)$ & + & + & + & + \\
$\operatorname{sign}\left(g_{4}(y)\right)$ & - & - & + & + \\
$\operatorname{sign}\left(g_{5}(y)\right)$ & + & + & + & + \\
\hline$V(y)$ & 5 & 4 & 1 & 0 \\
\hline & & & &
\end{tabular}


$g_{0}(y)$ has five real roots, so $V(-\infty)$ and $V(+\infty)$ are determined as in Table 1 . We also compute $V\left(\left(-B+\sqrt{B^{2}-4 C}\right) / 2\right)$ and $V\left(\left(-B-\sqrt{B^{2}-4 C}\right) / 2\right)$. Then we have

$$
\begin{aligned}
& V\left(\left(-B+\sqrt{B^{2}-4 C}\right) / 2\right)-V(\infty)=1 \\
& V\left(\left(-B-\sqrt{B^{2}-4 C}\right) / 2\right)-V\left(\left(-B+\sqrt{B^{2}-4 C}\right) / 2\right)=3, \\
& V(-\infty)-V\left(\left(-B-\sqrt{B^{2}-4 C}\right) / 2\right)=1
\end{aligned}
$$

Hence polynomial $(61)$ has one, three and one real roots in the intervals $((-B+$ $\left.\left.\sqrt{B^{2}-4 C}\right) / 2, \infty\right),\left(\left(-B-\sqrt{B^{2}-4 C}\right) / 2,\left(-B+\sqrt{B^{2}-4 C}\right) / 2\right)$ and $(-\infty,(-B-$ $\left.\sqrt{B^{2}-4 C}\right) / 2$ ), respectively.

Next we will analyze the local phase portraits of the finite equilibria. For this we first study the infinite equilibrium points. The infinite equilibrium points in the Poincaré sphere are the origin of $U_{1}$, and also the corresponding origin of $V_{1}$. The origins of $U_{1}$ and $V_{1}$ consist of six hyperbolic sectors when $C<B^{2} / 4$, hence, by Theorem 2.2, they have index -4 . In the Poincaré disk the finite equilibrium points must have total index 3 . Thus the finite equilibrium points must be four centers and one saddle. The only possible phase portrait of system (45) in this case is topologically equivalent to 1.20 of Figure 1.

(ii.a.2) Now we study the case when (61) has four distinct real roots. According to Theorem 2.1 the unique revised sign list of the discriminant sequence must be $[1,1,1,1,0]$, which needs $D_{1,2,3,4}>0$ and $D_{5}=0$. From the above case (ii.a.1) it is easy to get that $B>0, C<B^{2} / 4,0<\Delta<F_{4}$ and $D_{5}=0$. In the Poincaré disk the total index at the finite equilibria must be 3 . Hence the finite equilibrium points must have three centers and one cusp. The phase portrait of system (45) is topologically equivalent to 1.21 of Figure 1.

(ii.a.3) We will analyze the case when (61) has three distinct real roots. From Theorem 2.1 the revised sign list must be either $[1,1,1,0,0]$, or $[1,1,1,1,-1]$, or $[1,1,1,-1,-1]$, or $[1,1,-1,-1,-1]$, or $[1,-1,-1,-1,-1]$.

Assume that the revised sign list is $[1,1,1,0,0]$, it needs $D_{1,2,3}>0$ and $D_{4}=$ $D_{5}=0$. Then we obtain that $B=0, C<0$ and $\Delta=C^{2} / 2$.

If the revised sign lists are $[1,1,1,1,-1]$ and $[1,1,1,-1,-1]$, the associated discriminant sequence of the former is $[1,1,1,1,-1]$, and the later is $[1,1,1,-1,-1]$ or $[1,1,1,0,-1]$. Hence it just needs $D_{1,2,3}>0$ and $D_{5}<0$. Then we have $B=0$, $C<0, \Delta>C^{2} / 2$, or $B>0, C<B^{2} / 4, \Delta>0$ and $D_{5}<0$, or $B^{2} / 4<C<3 B^{2} / 10$ and $\Delta>F_{3}$.

If the revised sign list is $[1,1,-1,-1,-1]$ the associated discriminant sequences are $[1,1,-1,-1,-1],[1,1,0,-1,-1]$ and $[1,1,0,0,-1]$. From the first two discriminant sequences, we have that $B^{2} / 4<C<3 B^{2} / 10, F_{4}<\Delta \leq F_{3}$ and $D_{5}<0$. In addition we compute the resultant of $D_{3}$ and $D_{4}$ with respect to $B$ and obtain

$$
\text { Resultant }\left[D_{3}, D_{4}, B\right]=5308416 \Delta^{4}\left(10 \Delta+C^{2}\right)^{2}\left(16 \Delta+C^{2}\right)^{4} \neq 0 .
$$

Hence we have that the discriminant sequence $[1,1,0,0,-1]$ cannot be satisfied.

Similarly, if the revised sign list is $[1,-1,-1,-1,-1]$, we have that $B=0, C>0$ and $\Delta>C^{2} / 2$, or $B>0, C \geq 3 B^{2} / 10, \Delta>F_{4}$ and $D_{5}<0$. 
From the above analyzing we have $B=0, C<0$ and $\Delta \geq C^{2} / 2$, or $B=0$, $C>0$ and $\Delta>C^{2} / 2$, or $B>0, C<B^{2} / 4, \Delta>0$ and $D_{5}<0$, or $C>B^{2} / 4>0$, $\Delta>F_{4}$ and $D_{5}<0$ when systems (45) and (56) have three equilibria which are not in the set $L$. If $C<B^{2} / 4$ the origins of $U_{1}$ and $V_{1}$ consist of six hyperbolic sectors, which have index -4 . In the Poincaré disk the finite equilibrium points must have total index 3. Therefore the three finite equilibrium points must be centers. In this subcase we have the phase portrait 1.22 of Figure 1.

When $C>B^{2} / 4$ the origins of $U_{1}$ and $V_{1}$ consist of two hyperbolic sectors which have index 0 by Theorem 2.2. Thus in the Poincaré disk the remaining finite equilibria must have total index 1 . Hence the three finite equilibrium points must be two centers and one saddle. Then we have that phase portrait is topologically equivalent to 1.23 of Figure 1.

(ii.a.4) Assume that (61) has two distinct real roots, the revised sign list of the discriminant sequence must be $[1,1,0,0,0]$ or $[1,1,1,-1,0]$ or $[1,1,-1,-1,0]$ or $[1,-1,-1,-1,0]$.

If the revised sign list is $[1,1,0,0,0]$, it needs $D_{3}=D_{4}=D_{5}=0$ which cannot be satisfied. Because we have the resultants

$$
\begin{aligned}
\operatorname{Resultant}\left[D_{3}, D_{4}, C\right]= & -21233664 \Delta^{6} B^{4}\left(576 \Delta+B^{4}\right)^{2}, \\
\operatorname{Resultant}\left[D_{3}, D_{5}, C\right]= & -12230590464 \Delta^{10}\left(35184372088832 \Delta^{5}\right. \\
& +1745904205824 \Delta^{4} B^{4}+25686712320 \Delta^{3} B^{8} \\
& \left.+108235835 \Delta^{2} B^{12}+350104 \Delta B^{16}+432 B^{20}\right) .
\end{aligned}
$$

Clearly the equations $D_{3}=D_{4}=D_{5}=0$ have no common solutions in the case $\Delta>0$. On the other hand, there is no solutions with the parameters for the revised sign list $[1,1,1,-1,0]$.

If the revised sign list is $[1,1,-1,-1,0]$, its discriminant sequence is $[1,1,-1,-1,0]$ or $[1,1,0,-1,0]$. It just needs $D_{2}>0, D_{3} \leq 0, D_{4}<0$ and $D_{5}=0$. Hence we have $B^{2} / 4<C<3 B^{2} / 10, F_{4}<\Delta \leq F_{3}$ and $D_{5}=0$.

Assume that the revised sign list is $[1,-1,-1,-1,0]$, whose discriminant sequence is $[1,0,0,-1,0]$ or $[1,0,-1,-1,0]$ or $[1,-1,-1,-1,0]$. From the equations $D_{2}=D_{3}=0$ we have $B=C=0$, which cannot be satisfied. If $D_{2} \leq 0$ and $D_{3,4}<0$ we have either $B=0, C>0$ and $\Delta>C^{2} / 2$, or $B>0, C \geq 3 B^{2} / 10$ and $\Delta>F_{4}$. If $B=0, C>0$ and $\Delta>C^{2} / 2$, then we have $D_{5}=-1024 \Delta^{2}\left(2 \Delta-C^{2}\right)^{3}<0$, which also cannot be satisfied with $D_{5}=0$. Hence we have $C \geq 3 B^{2} / 10>0, \Delta>F_{4}$ and $D_{5}=0$.

In short systems (45) and (56) have two equilibria, which are not in the set $L$, if and only if $C>B^{2} / 4>0, \Delta>F_{4}$ and $D_{5}=0$. In this subcase the origins of $U_{1}$ and $V_{1}$ consist of two hyperbolic sectors which have index 0 . In the Poincaré sphere the finite equilibrium points must have total index 2. Hence the finite equilibrium points must be one center and one cusp. The phase portrait is topologically equivalent to 1.24 of Figure 1.

(ii.a.5) Now we study the case when the polynomial (61) has one distinct real root. For the sake of simplicity, we obtain the possible revised sign lists and their associated discriminant sequences as it is shown in the Table 2. We denote by RSL 
and DS the revised sign lists and the discriminant sequences in the following tables. In summary systems (45) and (56) have one equilibria, which is not in the set $L$, if and only if $B=0, C>0$ and $0<\Delta \leq C^{2} / 2$, or $C>B^{2} / 4>0, \Delta>0$ and $D_{5}>0$.

TABLE 2. The conditions of the revised sign lists.

\begin{tabular}{|c|c|c|}
\hline$\overline{\mathrm{RSL}}$ & $\overline{\mathrm{DS}}$ & Conditions \\
\hline \multirow[t]{4}{*}[1,-1,1,1,1]{} & {$[1,-1,1,1,1]$} & no solutions; \\
\hline & {$[1,-1,0,1,1]$} & no solutions; \\
\hline & {$[1,-1,0,0,1]$} & no solutions; \\
\hline & {$[1,0,1,1,1]$} & no solutions; \\
\hline \multirow[t]{3}{*}[1,1,-1,1,1]{} & {$[1,1,-1,1,1]$} & $\frac{B^{2}}{4}<C<\frac{3 B^{2}}{10}, 0<\Delta<F_{4}$ \\
\hline & {$[1,1,0,1,1]$} & no solutions; \\
\hline & {$[1,1,-1,0,1]$} & $\frac{B^{2}}{4}<C<\frac{3 B^{2}}{10}, \Delta=F_{4}$ \\
\hline \multirow{2}{*}[1,1,1,-1,1]{} & {$[1,1,1,-1,1]$} & no solutions; \\
\hline & {$[1,1,1,0,1]$} & no solutions; \\
\hline \multirow[t]{6}{*}[1,-1,-1,1,1]{} & {$[1,-1,-1,1,1]$} & $C>\frac{3 B^{2}}{10}, 0<\Delta<F_{4}$ \\
\hline & {$[1,0,-1,1,1]$} & $C=\frac{3 B^{2}}{10}>0,0<\Delta<\frac{(3 \sqrt{129}-21) B^{4}}{8000}$ \\
\hline & {$[1,0,-1,0,1]$} & $C=\frac{3 B^{2}}{10}>0, \Delta=\frac{(3 \sqrt{129}-21) B^{4}}{8000} ;$ \\
\hline & {$[1,0,0,1,1]$} & no solutions; \\
\hline & {$[1,0,0,0,1]$} & no solutions; \\
\hline & {$[1,-1,-1,0,1]$} & $C>\frac{3 B^{2}}{10}>0, \Delta=F_{4}$ \\
\hline \multirow[t]{3}{*}[1,1,-1,-1,1]{} & {$[1,1,-1,-1,1]$} & $\frac{B^{2}}{4}<C<\frac{3 B^{2}}{10}, \Delta>F_{4}, D_{5}>0$ \\
\hline & {$[1,1,0,-1,1]$} & no solutions; \\
\hline & {$[1,1,0,0,1]$} & no solutions; \\
\hline \multirow[t]{3}{*}[1,-1,-1,-1,1]{} & {$[1,-1,-1,-1,1]$} & $C>\frac{3 B^{2}}{10}>0, \Delta>F_{4}, D_{5}>0$ \\
\hline & {$[1,0,-1,-1,1]$} & $\begin{array}{l}C=\frac{3 B^{2}}{10}>0, \Delta>\frac{(3 \sqrt{129}-21) B^{4}}{8000} \\
D_{5}>0\end{array}$ \\
\hline & {$[1,0,0,-1,1]$} & no solutions. \\
\hline$[1,1,-1,0,0]$ & {$[1,1,-1,0,0]$} & no solutions; \\
\hline \multirow[t]{2}{*}[1,-1,-1,0,0]{} & {$[1,-1,-1,0,0]$} & $B=0, C>0, \Delta=C^{2} / 2$ \\
\hline & {$[1,0,-1,0,0]$} & no solutions. \\
\hline
\end{tabular}

The origins of $U_{1}$ and $V_{1}$ have two hyperbolic sectors, by Theorem 2.2, have index 0 . In the Poincaré sphere, the finite equilibrium points must have total index 2. Thus the finite equilibrium point must be a center. In this subcase we have the phase portrait 1.25 of Figure 1.

(ii.a.6) Now we study the case when equation (54) has no real roots. Similarly to the above case we have the revised sign lists $[1,-1,0,0,0],[1,-1,1,1,0]$ and $[1,1,-1,1,0]$. 


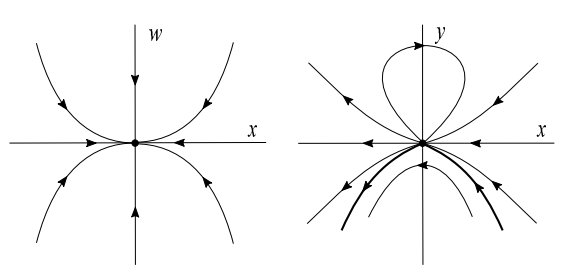

(a)

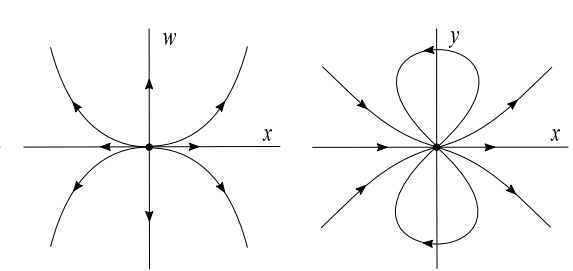

(b)

Figure 33. Blow-up at the origin of systems (56) and (67) with $\Delta=C=0$. (a) Systems (66) and (56), (b) Systems (68) and (67).

From the case (ii.a.4) we have $[1,-1,0,0,0]$ can not be satisfy. If the revised sign list is $[1,-1,1,1,0]$, the associated discriminant sequences are $[1,-1,1,1,0]$, $[1,0,1,1,0]$ and $[1,-1,0,1,0]$, it needs $D_{2} \leq 0$ and $D_{3} \geq 0$. From $D_{2} \leq 0$ we get that $C \geq 3 B^{2} / 10$, then have $D_{3} \leq\left. D_{3}\right|_{B^{2}=10 C / 3}=-8 C^{3} / 3<0$. Hence this case cannot be satisfied.

And it is easy to check that there is no solutions for revised sign list $[1,1,-1,1,0]$. Hence systems (45) and (56) have at least one equilibrium point, which is not in the set $L$, in the case $C \neq B^{2} / 4$ and $\Delta>0$.

By the continuity of the phase portraits from 1.20 to 1.22 , we use the bifurcation curve $\Delta=f_{4}(B, C)$ to describe the phase portrait 1.21. In addition, by the condition $\left.D_{5}\right|_{C=B^{2} / 4}=-\Delta^{4}\left(8192 \Delta+27 B^{4}\right)$, we obtain the coordinates of the intersection point $\left(\Delta, B^{2}-4 C\right)=(0,0)$ between the curve $\Delta=f_{4}(B, C)$ and $B^{2}-4 C=0$. Hence we have the phase portraits 1.20 and 1.22 when $\Delta>f_{4}(B, C)$ and $0<\Delta<f_{4}(B, C)$, respectively. Similarly we obtain the bifurcation curve $\Delta=$ $f_{5}(B, C)$ to describe the phase portrait 1.24. And in the intervals $0<\Delta<f_{5}(B, C)$ and $\Delta>f_{5}(B, C)$, it provides the phase portraits 1.23 and 1.25 , respectively, as it is shown in Figure 8.

Now we study the dynamics of system (56) in the case (ii.b), i.e. $\Delta=0$.

(ii.b.1) If $C>B^{2} / 4$ system (56) has one equilibrium point $p_{1}=(0,0)$, which is a center. In this subcase we have that the phase portrait is topologically equivalent to 1.25 of Figure 2.

(ii.b.2) If $C=0$ and $B \neq 0$ system (56) has two equilibrium points $p_{1}=(0,0)$ and $p_{2}=(0,-B)$, which are in the set $L$ and are linearly zero. To understand the local phase portrait of the origin we need to do the blow-up $(x, y) \rightarrow(x, w)$ with $w=y / x$, and we get the system

$$
\begin{aligned}
x^{\prime} & =x\left(-B-2 w x+B^{2} w^{3} x+2 B w^{4} x^{2}+w^{5} x^{3}\right), \\
w^{\prime} & =-w\left(B+B^{2} w^{3} x+2 B w^{4} x^{2}+w^{5} x^{3}\right),
\end{aligned}
$$

after eliminating the common factor $x$ between $x^{\prime}$ and $w^{\prime}$. When $x=0$ system (66) has one equilibrium located at the origin, which is an attracting node. Going back through the change of variables to the origin of system (56) and taking into account the behavior of the flow on the axes, it has one hyperbolic sector, one elliptic sector and two parabolic sectors, see Figure 33(a). 


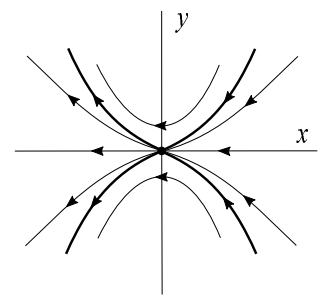

Figure 34. Blow-up at $p_{2}$ of system (56) with $\Delta=0$ and $0<C<B^{2} / 4$.

To determine the local behavior of $p_{2}$, we translate this equilibrium point to the origin doing the change of variables $x \rightarrow x$ and $y \rightarrow y-B$, we have the system

$$
x^{\prime}=B x^{2}-2 x^{2} y-B^{3} y^{2}+3 B^{2} y^{3}-3 B y^{4}+y^{5}, \quad y^{\prime}=2 x(B-y) y .
$$

Now we do the blow-up $(x, y) \rightarrow(x, w)$ with $w=y / x$, and eliminating the common factor $x$ between $x^{\prime}$ and $w^{\prime}$ we have

$$
\begin{aligned}
x^{\prime} & =-x\left(-B+B^{3} w^{2}+2 w x-3 B^{2} w^{3} x+3 B w^{4} x^{2}-w^{5} x^{3}\right), \\
w^{\prime} & =-w\left(-B-B^{3} w^{2}+3 B^{2} w^{3} x-3 B w^{4} x^{2}+w^{5} x^{3}\right) .
\end{aligned}
$$

The origin of system (68) is a repelling node. Going back through the change of variables we get that the origin of system (67) consists of two elliptic and two parabolic sectors (see Figure 33(b)). Going back to the associated Hamiltonian system (45) the equilibrium points $p_{1,2}$ are two virtual equilibrium points. Therefore the phase portrait in this case is topologically equivalent to 1.26 of Figure 2.

(ii.b.3) If $C<B^{2} / 4$ and $C \neq 0$ system (56) has three equilibrium points $p_{1}=$ $(0,0)$ and $p_{2,3}=\left(0,\left(-B \pm \sqrt{B^{2}-4 C}\right) / 2\right)$. The equilibrium points $p_{2,3}$ are in the set $L$ and they are linearly zero. The local phase portrait of $p_{2}$ consists of two elliptic sectors and two parabolic sectors, or two hyperbolic sectors and two parabolic sectors when $C<0$ or $C>0$ respectively (see Figures 33(b) and 34). The local phase portrait of $p_{3}$ consists of two elliptic and two parabolic sectors, see Figure 33(b). On the other hand, the equilibrium point $p_{1}$ is a saddle and a center when $C<0$ and $C>0$, respectively. Going back to the Hamiltonian system (45) the equilibrium points $p_{2,3}$ are two virtual equilibrium points. Hence we have the phase portraits 1.27 and 1.28 of Figure 2 when $C<0$ and $0<C<B^{2} / 4$.

(ii.c) Finally we study the dynamics of system (56) in the case $\Delta<0$. Similarly to the case (ii.a), we know that the equilibrium point $\left(0, y_{0}\right)$ of system $(56)$ is either a saddle or a center or a cusp. And system (56) has possible four equilibrium points

$$
\begin{aligned}
& p_{1,2}=\left(\sqrt{-\Delta},\left(-B \pm \sqrt{B^{2}-4 C}\right) / 2\right), \\
& p_{3,4}=\left(-\sqrt{-\Delta},\left(-B \pm \sqrt{B^{2}-4 C}\right) / 2\right),
\end{aligned}
$$

which are in the set $L$. The equilibrium points $p_{1,4}$ are attracting nodes, $p_{2,3}$ are repelling nodes. Now we consider the finite equilibria which are not in the set $L$.

Assume that $B \geq 0, \Delta<0$ and $C \neq B^{2} / 4$, from $D_{2}>0$ and $D_{3}>0$ we have $C<B^{2} / 4, C \neq 0$ and $F_{3}<\Delta<0$. From $D_{4}=0$ we obtain $\Delta=0$ or $\Delta=F_{4}$ or 
$\Delta=F_{6}$ where

$$
F_{6}=\frac{45 B^{4}-326 B^{2} C+512 C^{2}}{2560}-\frac{3 \sqrt{F_{5}}}{2560} .
$$

Here we have $F_{6}<F_{3}<0<F_{4}$. Because $\left.D_{4}\right|_{\Delta=0}=0$ and

$$
\left.D_{4}\right|_{\Delta=F_{3}}=-\frac{9 B^{2}\left(B^{2}-6 C\right)^{2}\left(B^{2}-4 C\right)^{2} C^{4}}{8\left(3 B^{2}-10 C\right)^{3}}<0,
$$

we get $D_{4}<0$. Therefore the polynomial (61) has at most three distinct roots. Similarly to the case (ii.a) the polynomial (61) has at least one distinct root.

(ii.c.1) If (61) has three distinct roots, the revised sign list must be $[1,1,1,0,0]$, or $[1,1,1,1,-1]$, or $[1,1,1,-1,-1]$, or $[1,1,-1,-1,-1]$, or $[1,-1,-1,-1,-1]$. From the above analysis we have that the revised sign list can not be $[1,1,1,0,0]$ and $[1,1,1,1,-1]$, because we have $D_{4}<0$ from $D_{2}>0$ and $D_{3}>0$. From Table 3 we have that systems (45) and (56) have three equilibria, which are not in the set $L$, if and only if $B>0,0<C, C \neq B^{2} / 4, F_{6}<\Delta<0$ and $D_{5}<0$.

TABLE 3. The conditions of the revised sign lists.

\begin{tabular}{lll}
\hline RSL & DS & Conditions \\
\hline$[1,1,1,-1,-1]$ & {$[1,1,1,-1,-1]$} & $0<C<\frac{B^{2}}{4}, F_{3}<\Delta<0, D_{5}<0 ;$ \\
& {$[1,1,1,0,-1]$} & no solutions; \\
\hline$[1,1,-1,-1,-1]$ & {$[1,1,-1,-1,-1]$} & $B>0,0<C<\frac{B^{2}}{4}, C \neq \frac{B^{2}}{6}$, \\
& & $F_{6}<\Delta<F_{3}, D_{5}<0$, or \\
& $\frac{B^{2}}{4}<C<\frac{3 B^{2}}{10}, F_{6}<\Delta<0, D_{5}<0 ;$ \\
& $B>0,0<C<\frac{B^{2}}{4}, C \neq \frac{B^{2}}{6}$, \\
& {$[1,1,0,-1,-1]$} & $\Delta=F_{3}, D_{5}<0 ;$ \\
& no solutions; \\
\hline$[1,-1,-1,-1,-1]$ & {$[1,-1,0,0,-1]$} & $C>\frac{3 B^{2}}{10}>0, F_{6}<\Delta<0, D_{5}<0 ;$ \\
& {$[1,0,-1,-1,-1]$} & $C=\frac{3 B^{2}}{10}>0, D_{5}<0$, \\
& $-\frac{(3 \sqrt{129}+21) B^{4}}{8000}<\Delta<0 ;$ \\
& no solutions. \\
\hline
\end{tabular}

Assume that $B^{2} / 4<C$ then the origins of $U_{1}$ and $V_{1}$ consist of two hyperbolic sectors which have index 0 by Theorem 2.2. Thus, in the Poincaré disk, the finite equilibrium points must have total index 1 . Hence the finite equilibrium points must be two centers and one saddle. The phase portrait is topologically equivalent to 1.23 of Figure 1.

The origins of $U_{1}$ and $V_{1}$ have six hyperbolic sectors when $C<B^{2} / 4$, hence, by Theorem 2.2, have index -4 . And the known equilibrium points $p_{1,2,3,4}$ have total index 4. Thus the remaining finite equilibrium points must have total index -1 in Poincaré disk. Then the remaining finite equilibrium points must be one center and two saddles. Going back to the Hamiltonian system (45), the equilibria $p_{1,2,3,4}$ are four virtual equilibrium points, therefore we obtain that the phase portrait of system (45) is topologically equivalent to 1.29 of Figure 2. 
TABLE 4. The conditions of the revised sign lists.

\begin{tabular}{|c|c|c|}
\hline RSL & $\mathrm{DS}$ & Conditions \\
\hline \multirow[t]{4}{*}[1,-1,1,1,1]{} & {$[1,-1,1,1,1]$} & $C>\frac{3 B^{2}}{10}, \Delta<F_{3}$ \\
\hline & {$[1,-1,0,1,1]$} & $C>\frac{3 B^{2}}{10}>0, \Delta=F_{3}$ \\
\hline & {$[1,-1,0,0,1]$} & $B=0, C>0, \Delta=-\frac{C^{2}}{10}$ \\
\hline & {$[1,0,1,1,1]$} & no solutions; \\
\hline \multirow[t]{4}{*}[1,1,-1,1,1]{} & {$[1,1,-1,1,1]$} & $C<\frac{3 B^{2}}{10}, \Delta<F_{6}$ \\
\hline & {$[1,1,0,1,1]$} & no solutions; \\
\hline & {$[1,1,-1,0,1]$} & $B>0, C<\frac{3 B^{2}}{10}, C \neq 0, C \neq \frac{B^{2}}{6}$, \\
\hline & & $\Delta=F_{6}$ \\
\hline \multirow[t]{2}{*}[1,1,1,-1,1]{} & {$[1,1,1,-1,1]$} & $C<\frac{B^{2}}{4}, F_{3}<\Delta<0, C \neq 0, D_{5}>0$ \\
\hline & {$[1,1,1,0,1]$} & no solutions; \\
\hline \multirow[t]{6}{*}[1,-1,-1,1,1]{} & {$[1,-1,-1,1,1]$} & $C>\frac{3 B^{2}}{10}>0, F_{3}<\Delta<F_{6}$ \\
\hline & {$[1,-1,-1,0,1]$} & $C>\frac{3 B^{2}}{10}>0, \Delta=F_{6}, C \neq \frac{5 B^{2}}{16}$ \\
\hline & {$[1,0,-1,1,1]$} & $C=\frac{3 B^{2}}{10}>0, \Delta<-\frac{(3 \sqrt{129}+21) B^{4}}{8000} ;$ \\
\hline & {$[1,0,-1,0,1]$} & $C=\frac{3 B^{2}}{10}>0, \Delta=-\frac{(3 \sqrt{129}+21) B^{4}}{8000} ;$ \\
\hline & {$[1,0,0,1,1]$} & no solutions; \\
\hline & {$[1,0,0,0,1]$} & no solutions; \\
\hline \multirow[t]{6}{*}[1,1,-1,-1,1]{} & {$[1,1,-1,-1,1]$} & either $B>0, C<\frac{B^{2}}{4}, C \neq 0, C \neq \frac{B^{2}}{6}$, \\
\hline & & $F_{6}<\Delta<F_{3}, D_{5}>0$, or \\
\hline & & $\frac{B^{2}}{4}<C<\frac{3 B^{2}}{10}, F_{6}<\Delta<0, D_{5}>0$ \\
\hline & {$[1,1,0,-1,1]$} & $\begin{array}{l}B>0, C<\frac{B^{2}}{4}, C \neq \frac{B^{2}}{6}, C \neq 0 \\
\Delta=F_{3}, D_{5}>0\end{array}$ \\
\hline & {$[1,1,0,0,1]$} & either $B=0, C<0, \Delta=-\frac{C^{2}}{10}$, \\
\hline & & or $B>0, C=\frac{B^{2}}{6}, \Delta=F_{3}=F_{6}$ \\
\hline \multirow[t]{4}{*}[1,-1,-1,-1,1]{} & {$[1,-1,-1,-1,1]$} & $C>\frac{3 B^{2}}{10}, F_{6}<\Delta<0, D_{5}>0$ \\
\hline & {$[1,0,-1,-1,1]$} & $C=\frac{3 B^{2}}{10}>0,-\frac{(3 \sqrt{129}+21) B^{4}}{8000}<\Delta<0$ \\
\hline & & $D_{5}>0$ \\
\hline & {$[1,0,0,-1,1]$} & no solutions; \\
\hline$[1,1,-1,0,0]$ & {$[1,1,-1,0,0]$} & no solutions; \\
\hline \multirow[t]{2}{*}[1,-1,-1,0,0]{} & {$[1,-1,-1,0,0]$} & $C=\frac{5 B^{2}}{16}>0, \Delta=F_{6}$ \\
\hline & {$[1,0,-1,0,0]$} & no solutions. \\
\hline
\end{tabular}

(ii.c.2) If the polynomial (61) has two distinct roots, the revise sign list must be $[1,1,1,-1,0]$ or $[1,1,-1,-1,0]$ or $[1,-1,-1,-1,0]$. Similarly to the case (ii.c. 1 ) we obtain that the condition is $B>0,0<C, C \neq B^{2} / 4, F_{6}<\Delta<0$ and $D_{5}=0$. 
When $B^{2} / 4<C$ the phase portrait of system (45) is topologically equivalent to 1.24 of Figure 1. If $C<B^{2} / 4$ system (56) has more four equilibrium points $p_{1,2,3,4}$, which have total index 4 . Hence the remaining finite equilibrium points must be one cusp and one saddle so that their index is -1 . Going back to system (45), the equilibrium points $p_{1,2,3,4}$ are four virtual equilibria. Hence the phase portrait is topologically equivalent to 1.30 of Figure 2.

(ii.c.3) If the polynomial (61) has one distinct root, we obtain the revise sign lists as it is shown in Table 4 . Then we have $B=0, C \neq 0$ and $\Delta<0$, or $B>0$, $C<B^{2} / 4$ and $\Delta<F_{6}$, or $B>0, C<B^{2} / 4, C \neq 0, F_{6} \leq \Delta<0$ and $D_{5}>0$, or $C>B^{2} / 4>0, \Delta<0$ and $D_{5}>0$.

When $B^{2} / 4<C$ the phase portrait of system (45) is topologically equivalent to 1.25 of Figure 1. If $C<B^{2} / 4$ the known infinite and finite equilibrium points have total index 4 . Hence the remaining finite equilibrium points must be one saddle so that it has index -1 . Going back to the Hamiltonian system (45) we have the phase portrait 1.31 of Figure 2.

In the case $\Delta<0$, for the sake of simplicity, we obtain the bifurcation curve $\Delta=$ $f_{6}(B, C)$ which provides the phase portrait 1.24 . By $\left.D_{5}\right|_{C=B^{2} / 4}=-\Delta^{4}(8192 \Delta+$ $\left.27 B^{4}\right)=0$, we obtain the coordinates of the intersection points $\left(\Delta, B^{2}-4 C\right)=$ $(0,0)$ and $\left(\Delta, B^{2}-4 C\right)=\left(-27 B^{4} / 8192,0\right)$ between the curve $\Delta=f_{6}(B, C)$ and $B^{2}-4 C=0$. Therefore we have the intervals $f_{6}(B, C)<\Delta<0$ and $\Delta<f_{6}(B, C)$ which describe the phase portraits 1.23 and 1.25 , respectively. Similarly we have the bifurcation curve $\Delta=f_{7}(B, C)$ which provides the phase portrait 1.30. And in the intervals $f_{7}(B, C)<\Delta<0$ and $\Delta<f_{7}(B, C)$, we have the phase portraits 1.29 and 1.31 respectively, as it is shown in Figure 8. Therefore we have concluded the proof of Theorem 1.5.

\section{ACKNOWLEDGMENTs}

The first author is partially supported by National Natural Science Foundation of China (No. 11771059) and China Scholarship Council (No. 201706130102). The second author is partially supported by the MINECO-FEDER grant MTM201677278-P, the AGAUR grant 2017 SGR1617, and the European project DynamicsH2020-HSCA-RISE-2017-777911.

\section{REFERENCES}

[1] M. J. Álvarez, A. Ferragut, X. Jarque, A survey on the blow up technique, Int. J. Bifurcation Chaos 31, (2011) 3103-3118.

[2] J. Artés, J. Llibre, Quadratic Hamiltonian vector fields, J. Differential Equations 107 (1994) $80-95$.

[3] T. R. Blows, C. Rousseau, Bifurcation at infinity in polynomial vector fields, J. Differential Equations 104 (1993) 215-242.

[4] T. Chen, L. Huang, P. Yu, W. Huang, Bifurcation of limit cycles at infinity in piecewise polynomial systems, Nonlinear Anal. 41 (2018) 82-106.

[5] I. Colak, J. Llibre, C. Valls, Hamiltonian linear type centers of linear plus cubic homogeneous polynomial vector fields, J. Differential Equations 257 (2014) 1623-1661.

[6] I. Colak, J. Llibre, C. Valls, Hamiltonian nilpotent centers of linear plus cubic homogeneous polynomial vector fields, Adv. Math. 259 (2014) 655-687. 
[7] I. Colak, J. Llibre, C. Valls, Bifurcation diagrams for Hamiltonian linear type centers of linear plus cubic homogeneous polynomial vector fields, J. Differential Equations 258 (2015) 846-879.

[8] I. Colak, J. Llibre, C. Valls, Bifurcation diagrams for Hamiltonian nilpotent centers of linear plus cubic homogeneous polynomial vector fields, J. Differential Equations 262 (2017) 55185533.

[9] F. Dumortier, J. Llibre, J. Artés, Qualitative Theory of Planar Differential Systems, Universitext, Springer-Verlag, New York, 2006.

[10] A. Gasull, A. Guillamon, V. Mañosa, Phase portrait of Hamiltonian systems with homogeneous nonlinearities, Nonlinear Anal. 42 (2000) 679-707.

[11] A. Guillamon, C. Pantazi, Phase portraits of separable Hamiltonian systems, Nonlinear Anal. 74 (2011) 4012-4035.

[12] J. Llibre, R. D. S. Oliveira, Phase portraits of quadratic polynomial vector fields having a rational first integral of degree 3, Nonlinear Anal. 70 (2009) 3549-3560.

[13] Y. Martínez, C. Vidal, Classification of global phase portraits and bifurcation diagrams of Hamiltonian systems with rational potential, J. Differential Equations 261 (2016) 5923-5948.

[14] D. Schlomiuk, Algebraic particular integrals, integrability and the problem of the center, Trans. Amer. Math. Soc. 338 (1993) 799-841.

[15] D. Schlomiuk, N. Vulpe, Global topological classification of Lotka-Volterra quadratic differential systems, Electronic J. Differential Equations 64 (2012) 1-69.

[16] N. Vulpe, Affine-invariant conditions for topological distinction of quadratic systems in the presence of a center (Russian), Differ. Equ. 19 (1983) 371-379.

[17] L. Yang, Recent advances on determining the number of real roots of parametric polynomials, J. Symbolic Comput. 28 (1999) 225-242.

1 College of Mathematics and Econometrics, Hunan University, Changsha, 410082, PR CHINA

E-mail address: chenting0715@126.com

2 Departament de Matemàtiques, Universitat Autònoma de Barcelona, 08193 Bellaterra, Barcelona, Catalonia, Spain, This is the corresponding author

E-mail address: jllibre@mat.uab.cat 